\title{
Superscars for Arithmetic Toral Point Scatterers
}

\author{
Pär Kurlberg, Lior Rosenzweig \\ Department of Mathematics, KTH Royal Institute of Technology, 10044 Stockholm, Sweden. \\ E-mail: kurlberg@math.kth.se; liorr@math.kth.se
}

Received: 7 October 2015 / Accepted: 23 June 2016

Published online: 21 October 2016 - (C) The Author(s) 2016. This article is published with open access at Springerlink.com

\begin{abstract}
We investigate eigenfunctions of the Laplacian perturbed by a delta potential on the standard tori $\mathbb{R}^{d} / 2 \pi \mathbb{Z}^{d}$ in dimensions $d=2,3$. Despite quantum ergodicity holding for the set of "new" eigenfunctions we show that superscars occur-there is phase space localization along families of closed orbits, in the sense that some semiclassical measures contain a finite number of Lagrangian components of the form $c_{i} \cdot d x \delta\left(\xi-\xi_{i}\right)$, for $c_{i}>0$ uniformly bounded from below. In particular, for both $d=2$ and $d=3$, eigenfunctions fail to equidistribute in phase space along an infinite subsequence of new eigenvalues. For $d=2$, we also show that some semiclassical measures have both strongly localized momentum marginals and non-uniform quantum limits (i.e., the position marginals are non-uniform). For $d=3$, superscarred eigenstates are quite rare, but for $d=2$ we show that the phenomenon is quite common-with $N_{2}(x) \sim x / \sqrt{\log x}$ denoting the counting function for the new eigenvalues below $x$, there are $\gg N_{2}(x) / \log ^{A} x$ eigenvalues $\lambda$ with the property that any semiclassical limit along these eigenvalues exhibits superscarring.
\end{abstract}

\section{Introduction}

A basic question in Quantum Chaos is the classification of quantum limits of energy eigenstates of quantized Hamiltonians. For example, if the classical dynamics is given by the geodesic flow on a compact Riemannian manifold $M$, the quantized Hamiltonian is given by the positive Laplacian $-\Delta$ acting on $L^{2}(M)$. With $\left\{\psi_{\lambda}\right\}_{\lambda}$ denoting Laplace eigenfunctions giving an orthonormal basis for $L^{2}(M)$, a quantum limit is a weak* limit of $\left|\psi_{\lambda}(x)\right|^{2}$ along any subsequence of eigenvalues $\lambda$ tending to infinity. More generally, given a smooth observable, i.e., a smooth function $f$ on the unit cotangent bundle $S^{*}(M)$, its quantization is defined as a pseudo-differential operator $\operatorname{Op}(f)$, and

P. K. and L. R. were partially supported by grants from the Göran Gustafsson Foundation for Research in Natural Sciences and Medicine, and the Swedish Research Council (621-2011-5498). 
one wishes to understand possible semiclassical measures, i.e., limits of the Wigner distributions

$$
f \rightarrow\left\langle\mathrm{Op}(f) \psi_{\lambda}, \psi_{\lambda}\right\rangle
$$

on $C^{\infty}\left(S^{*}(M)\right)$, as $\lambda \rightarrow \infty$. If $M$ has negative curvature ("strong chaos"), the celebrated quantum unique ergodicity (QUE) conjecture by Rudnick and Sarnak [34] asserts that the only quantum limit is given by the uniform, or Liouville, measure on $S^{*}(M)$. Conversely, if the geodesic flow is integrable, many non-uniform quantum limits (and semiclassical measures) may exist. E.g., if $M=\mathbb{R}^{2} / 2 \pi \mathbb{Z}^{2}$ is a flat torus and $a \in \mathbb{Z}$, then $\psi_{a}(x, y)=$ $\cos (a x) \cos (y)$ is an eigenfunction with eigenvalue $a^{2}+1$, and clearly $\left|\psi_{a}(x, y)\right|^{2} \stackrel{*}{\rightarrow}$ $\cos ^{2}(y) / 2$ as $a \rightarrow \infty$. (For a partial classification of the set of quantum limits on $\mathbb{R}^{2} / 2 \pi \mathbb{Z}^{2}$, see [21]).

Now, if the flow is ergodic ("weak chaos"), Schnirelman's theorem $[8,40,46]$ asserts Quantum Ergodicity, namely that the only semiclassical measure, provided we remove a zero density subset of the eigenvalues, is the uniform one. However, non-uniform semiclassical measures may exist along the zero density subsequence of removed eigenvalues. Some interesting questions for quantum ergodic systems are thus: are there any non-uniform semiclassical measures? If so, how large can the exceptional set of eigenvalues be? Can eigenfunctions have non-uniform quantum limits, i.e., is it possible that $\left|\psi_{\lambda}(x)\right|^{2}$, along some subsequence, weakly tends to something other than $1 / \operatorname{vol}(M)$ ? We shall address these questions for the set of "new" eigenfunctions of the Laplacian on a torus perturbed by a delta potential. The perturbation has a very small effect on the classical dynamics - only a zero measure subset of the set of trajectories is changed (hence there is no classical ergodicity), yet, as was recently shown [27,35,45], quantum ergodicity holds for the set of new eigenfunctions. (We note that this is quite different from point scatterers on tori of the form $\mathbb{R}^{2} / \Gamma$, for $\Gamma$ a generic rectangular lattice. Here it was recently shown [26] that quantum ergodicity does not hold; in fact almost all new eigenfunction exhibit strong momentum localization, or "superscarring", cf. Sect. 1.2).

1.1. Toral point scatterers. The point scatterer, or the Laplacian perturbed with a delta potential (also known as a "Fermi pseudopotential"), is a popular "toy model" for studying the transition between chaos and integrability in quantum chaos. With $\mathbb{T}^{d}:=\mathbb{R}^{d} / 2 \pi \mathbb{Z}^{d}$ for $d=2$ or $d=3$, let $\alpha \in \mathbb{R}$ denote the "strength" of a delta potential placed at some point $x_{0} \in \mathbb{T}^{d}$; the formal operator

$$
-\Delta+\alpha \cdot \delta_{x_{0}}
$$

can then be realized using von Neumann's theory of self adjoint extensions. For $d=2,3$ there is a one parameter family of self adjoint extensions $H_{\varphi}$, parametrized by an angle $\varphi \in(-\pi, \pi]$, and the quantum dynamics we consider is generated by $H_{\varphi}$. For $d=3$ we will keep $\varphi$ fixed, but in order to obtain a strong spectral perturbation for $d=2$ we will allow $\varphi$ to slowly vary with the eigenvalue; in the physics literature this is known as the "strong coupling limit", cf. Sect. 2 for more details.

The spectrum of $H_{\varphi}$ consists of two types of eigenvalues: "old" and "new" eigenvalues. The old ones are eigenvalues of the unperturbed Laplacian, i.e., integers that can be represented as sums of $d$ integer squares, and the old eigenfunctions are the corresponding eigenfunctions of the unperturbed Laplacian that vanish at $x_{0}$. The set of 
new eigenvalues, denoted by $\Lambda$, are all of multiplicity 1 , and interlace between the old eigenvalues. In fact, the new eigenvalues are solutions of the spectral equation

$$
\sum_{n \in \mathcal{N}_{d}} r_{d}(n)\left(\frac{1}{n-\lambda}-\frac{n}{n^{2}+1}\right)=C,
$$

where

$$
r_{d}(n):=\sum_{v \in \mathbb{Z}^{d},|v|^{2}=n} 1
$$

is the number of ways to represent $n$ as a sum of $d$ squares,

$$
\mathcal{N}_{d}:=\left\{n \in \mathbb{Z}: r_{d}(n)>0\right\},
$$

and

$$
C=C(\varphi):=\tan (\varphi / 2) \cdot \sum_{n} r_{d}(n) /\left(n^{2}+1\right)
$$

is allowed to vary with $\lambda$ when $d=2$.

For $\lambda \in \Lambda$ a new eigenvalue, the corresponding eigenfunction is then given by the Green's functions $G_{\lambda}=(\Delta+\lambda)^{-1} \delta_{x_{0}}$, with $L^{2}$-expansion

$$
G_{\lambda}(x)=-\frac{1}{4 \pi^{2}} \sum_{v \in \mathbb{Z}^{d}} \frac{\exp \left(-i v \cdot x_{0}\right)}{|v|^{2}-\lambda} e^{i v \cdot x} .
$$

We remark that the delta potential introduces singularities at $x_{0}$; as $x \rightarrow x_{0}$, we have the asymptotic

$$
G_{\lambda}(x)= \begin{cases}a_{\lambda} \cdot\left(\cos (\varphi / 2) \cdot \frac{\log \left|x-x_{0}\right|}{2 \pi}+\sin (\varphi / 2)\right)+o(1) & \text { for } d=2, \\ a_{\lambda} \cdot\left(\cos (\varphi / 2) \cdot \frac{-1}{4 \pi\left|x-x_{0}\right|}+\sin (\varphi / 2)\right)+o(1) & \text { for } d=3,\end{cases}
$$

where $a_{\lambda} \in \mathbb{R}^{\times}$for $\lambda \notin \mathcal{N}_{d}$ (cf. [35], proof of Lemma A.6). Note that $\varphi=\pi$ gives the unperturbed Laplacian; in what follows we will assume that $\varphi \in(-\pi, \pi)$.

We can now formulate our first result, namely that some eigenfunctions strongly localize in the momentum representation in dimension three. For $l \in \mathcal{N}_{3} \backslash\{0\}$ let

$$
\Omega(l):=\left\{v /|v| \in \mathbb{S}^{2}: v \in \mathbb{Z}^{3},|v|^{2}=l\right\}
$$

be the projection of the lattice points of distance $\sqrt{l}$ from the origin onto the unit sphere, and let $\mu_{\Omega(l)}$ denote the distribution defined by

$$
\mu_{\Omega(l)}(f):=\frac{1}{r_{3}(l)} \sum_{\substack{v \in \mathbb{Z}^{3} \\|v|^{2}=l}} \int_{\mathbb{T}^{3}} f\left(x, \frac{v}{|v|}\right) d x, \quad \text { for } f \in C^{\infty}\left(S^{*}\left(\mathbb{T}^{3}\right)\right),
$$

(we use the identification $S^{*}\left(\mathbb{T}^{3}\right) \simeq \mathbb{T}^{3} \times \mathbb{S}^{2}$ ), and let $v$ denote the Liouville measure on $S^{*}\left(\mathbb{T}^{3}\right)$. 
Theorem 1. Let $\mathbb{T}^{3}=\mathbb{R}^{3} / 2 \pi \mathbb{Z}^{3}, x_{0} \in \mathbb{T}^{3}$ and let $\Lambda$ be the set of "new" eigenvalues of the point scatterer, that is $\Lambda=\operatorname{Spec}\left(\mathrm{H}_{\varphi}\right) \backslash \mathcal{N}_{3}$. For $\lambda \in \Lambda$, let $g_{\lambda} \in L^{2}\left(\mathbb{T}^{2}\right)$ denote the $L^{2}$ normalized eigenfunction with eigenvalue $\lambda$. Then for any $l \in \mathcal{N}_{3}$ there exists an infinite subset $\Lambda_{l} \subset \Lambda$, and $a \in\left[\frac{1}{2}, 1\right]$ such that for any smooth observable $f \in C^{\infty}\left(S^{*}\left(\mathbb{T}^{3}\right)\right)$,

$$
\lim _{\lambda \rightarrow \infty, \lambda \in \Lambda_{l}}\left\langle O p(f) g_{\lambda}, g_{\lambda}\right\rangle=a \cdot \mu_{\Omega(l)}(f)+(1-a) \cdot v(f) .
$$

We note that $\mu_{\Omega(l)}$ can be decomposed into a finite sum of components of the form $d x \delta\left(\xi-\xi_{i}\right) / r_{3}(l)$, where $\xi_{i}$ ranges over elements in $\Omega(l)$. Each such component is microlocalized on a Lagrangian plane $\left\{(x, \xi) \in S^{*}\left(\mathbb{T}^{3}\right): x \in \mathbb{T}^{3}, \xi=\xi_{i}\right\}$, which in turn can be viewed as a family of closed orbits in the direction $\xi_{i}$-sometimes called a superscar (cf. [7]). In contrast, Yesha has shown that quantum ergodicity holds for this model [45], and that the only quantum limit is the uniform one [44]; as we shall see, the latter is not true in dimension two.

Since $a \geq 1 / 2$ and $\mu_{\Omega(l)}$ is a probability measure, we also note that the singular part has measure at least $1 / 2$. In comparison, for Anasov systems we expect that semiclassical measures can have mass at most $1 / 2$ on a periodic orbit; for quantized cat maps this is shown in [12]. More generally, the upper bound also follows from certain entropy estimates [1, Conjecture 1.5] known to hold for geodesic flows on manifolds with constant sectional curvature - 1 , and for Anasov geodesic flows in dimension two [2,32].

In dimension 2, when $\varphi$ is fixed, (1) is often referred to as the "weak coupling limit", and almost all new eigenvalues remain close to the old eigenvalues (cf. [36]). In the physics literature another quantization, sometimes referred to as the "strong coupling limit", is considered to be more interesting. In [39], Shigehara introduced the notions of a bare, as well as a physical (or renormalized), coupling constant, and argued that "wave chaos" appears for fixed physical coupling constant (strong coupling), but not for fixed bare coupling constant (weak coupling). In particular, he numerically found level repulsion in the former, but not in the latter-interestingly, the level spacing distribution for the Šeba billiard does not seem to be given by random matrix theory (see [6] for an analytical derivation of the two point correlation, assuming a certain random model for the unperturbed spectrum).

A notable difference from the weak coupling limit, where the operator $H_{\varphi}$ is a fixed self adjoint extension, the self adjoint extension is allowed to vary with $\lambda$ in the strong coupling limit. One way to arrive at the strong coupling limit is to only consider energy levels in a window around a given eigenvalue: e.g., for $\eta \in(131 / 146,1)$ the new eigenvalues are defined to be solutions of

$$
\sum_{\substack{n \in \mathcal{N}_{2} \\ n_{+}(\lambda) \mid<n_{+}(\lambda)^{\eta}}} r_{d}(n)\left(\frac{1}{n-\lambda}-\frac{n}{n^{2}+1}\right)=0,
$$

where $n_{+}(\lambda)$ is the smallest element of $\mathcal{N}_{2}$ that is larger than $\lambda$. It is convenient to consider both couplings simultaneously; we may do this by letting

$$
F(\lambda)= \begin{cases}\text { Constant } & \text { (weak coupling) } \\ \sum_{\substack{n \in \mathcal{N}_{2} \\\left|n-n_{+}(\lambda)\right| \geq n_{+}(\lambda)^{\eta}}} r_{2}(n)\left(\frac{1}{n-\lambda}-\frac{n}{n^{2}+1}\right) & \text { (strong coupling) }\end{cases}
$$


and then rewriting the spectral equation as

$$
\sum_{n \in \mathcal{N}_{2}} r_{2}(n)\left(\frac{1}{n-\lambda}-\frac{n}{n^{2}+1}\right)=F(\lambda)
$$

We remark that $F(\lambda)=-\pi \log \lambda+O(1)$ in the strong coupling case, see [43, Section 3] for details.

Our next result, valid for both the weak and strong coupling limit in dimension two, is the existence of a zero density subsequence of eigenfunctions whose associated semiclassical measure have singular momentum marginal, as well as non-uniform position marginal.

Theorem 2. Let $\mathbb{T}^{2}=\mathbb{R}^{2} / 2 \pi \mathbb{Z}^{2}, x_{0} \in \mathbb{T}^{2}$ and let $\Lambda$ be the set of new eigenvalues of the point scatterer, that is $\Lambda=\operatorname{Spec}\left(\mathrm{H}_{\varphi}\right) \backslash \mathcal{N}_{2}$. For $\lambda \in \Lambda$, let $g_{\lambda} \in L^{2}\left(S^{*} \mathbb{T}^{2}\right)$ be the $L^{2}$-normalized eigenfunction with eigenvalue $\lambda$. There exists an infinite subset $\Lambda^{\prime} \subset \Lambda$ having a semiclassical limit

$$
v_{\infty}(f):=\lim _{\lambda \rightarrow \infty, \lambda \in \Lambda^{\prime}}\left\langle\mathrm{Op}(f) g_{\lambda}, g_{\lambda}\right\rangle
$$

such that $v_{\infty}$ is non-uniform in position, i.e., with $x=\left(x_{1}, x_{2}\right) \in \mathbb{T}^{2}$,

$$
v_{\infty}\left(e^{i \cdot 2 x_{2}}\right)=c>0
$$

and $v_{\infty}$ is strongly localized in momentum-for positive momentum observables $f(x, \xi)=f_{0}(\xi) \geq 0$,

$$
v_{\infty}(f) \geq \frac{1}{2} \mu_{\Omega(\infty)}(f)
$$

where $\Omega(\infty) \subset \mathbb{S}^{1}$ is a multiset of bounded cardinality, and

$$
\mu_{\Omega(\infty)}:=\frac{1}{|\Omega(\infty)|} \sum_{\xi \in \Omega(\infty)} \delta_{\xi}
$$

Note that $\mu_{\Omega(\infty)}$ is a probability measure, hence at least half the mass is carried on the singular part in the momentum representation.

In order to quantify how common localized eigenfunctions are we need some further notation. For $d=2,3$, let $N_{d}(x)$ denote the counting function ("Weyl's law") for the number of new eigenvalues $\lambda \leq x$. For $d=3, N_{3}(x) \sim x$ and, as the eigenvalues that give rise to superscars are essentially powers $4^{l}$, the exceptional subset is of size $x^{o(1)}$ and thus very sparse. For $d=2, N_{2}(x) \sim x / \sqrt{\log x}=x^{1-o(1)}$, and our construction of eigenfunctions that are non-uniform both in position and momentum gives a corresponding subset of eigenvalues with counting function of size at most $x^{1 / 2-o(1)}$ - hence fairly rare. However, if we restrict ourselves to non-uniformity only in the momentum representation, we can use some recent results by Maynard [31] to show that localized eigenfunctions are in fact quite common. 
Theorem 3. With notations as in Theorem 2, there exists a subset $\Lambda^{\prime \prime} \subset \Lambda$ with the following property: if $\Lambda^{\prime \prime \prime} \subset \Lambda^{\prime \prime}$ is any infinite subset such that the semiclassical limit

$$
v_{\infty}(f):=\lim _{\lambda \in \Lambda^{\prime \prime \prime}}\left\langle\mathrm{Op}(f) g_{\lambda}, g_{\lambda}\right\rangle
$$

exists, then $v_{\infty}$ is strongly localized in momentum in the sense that there exist a multiset $\Omega(\infty) \subset \mathbb{S}^{1}$, allowed to depend on $v_{\infty}$ but of uniformly bounded cardinality, such that for positive momentum observables $f(x, \xi)=f_{0}(\xi) \geq 0$,

$$
v_{\infty}(f) \geq c \cdot \mu_{\Omega(\infty)}(f),
$$

where $c>0$ is an absolute constant. Moreover,

$$
\left|\left\{\lambda \in \Lambda^{\prime \prime}: \lambda \leq x\right\}\right| \gg x /(\log x)^{A}
$$

for some $A>1$.

Remark 4. Without loss of asymptotic density, we may "shrink" $\Lambda$ " such that $v_{\infty}$ in addition is "flat in position", i.e., $v_{\infty}(f)=0$ for any observable $f$ such that $\int_{\mathbb{T}^{2}} f(x, \xi) d x=0$ for all $\xi \in \mathbb{S}^{1}$ (cf. Appendix A). Hence $\nu_{\infty}$ contains components that are microlocalized on Lagrangian planes, but unlike the case $d=3$ (cf. Theorem 1), we do not obtain Liouville measure on the complementary components.

1.2. Discussion. In [37] Šeba proposed quantum billiards on rectangles with irrational aspect ratio, perturbed with a delta potential, as a solvable singular model exhibiting wave chaos; in particular that the level spacings should be given by random matrix theory (GOE). Seba and Życzkowski later noted [38] that the level spacings were not consistent with GOE, in particular large gaps are much more frequent (essentially having a Poisson distribution tail). Shigehara subsequently found [39] that level repulsion is only present in the strong coupling limit. Recently Rudnick and Ueberschär proved [36], in dimension two, that the level spacing for the weak coupling limit is the same as the level spacings of the unperturbed Laplacian (after removing multiplicities). This in turn is conjectured to be Poissonian, and we note that a natural analogue of the prime $k$-tuple conjecture for integers that are sums of two squares can be shown to imply Poisson gaps [14]. In [36] the three dimensional case was also investigated and the mean displacement between new and old eigenvalues was shown to equal half the mean spacing.

In [35], Rudnick and Ueberschär proved a position space analogue of Quantum Ergodicity for the new eigenfunctions: there exists a full density subset of the new eigenvalues such that as $\lambda \rightarrow \infty$ along this subset, the only weak limit of $\left|\psi_{\lambda}(x)\right|^{2}$ is the uniform measure on $\mathbb{T}^{2}$. Further, in [27] the first author and Ueberschär proved an analogue of Quantum Ergodicity: there exists a full density subset of the new eigenvalues such that the only quantum limit along this subset is the uniform measure on the full phase space (i.e., the unit cotangent bundle $S^{*}\left(\mathbb{T}^{d}\right)$ ). This result was later shown to hold also for $d=3$ by Yesha [45]; already in [44] he showed that all eigenfunctions equidistribute in the position representation.

For irrational tori, Keating, Marklof and Winn proved [22] that there exist nonuniform quantum limits (in fact, strong momentum scarring was already observed in [5]), assuming a spectral clustering condition implied by the old eigenvalues having Poisson spacings (which in turn follows from the Berry-Tabor conjecture). Recently the first author and Ueberschär unconditionally showed [26] that for tori having diophantine 
aspect ratio, essentially all new eigenfunctions exhibit superscarring in the following sense: given any $\delta>0$, the proportion of eigenfunction exhibiting superscarring is at least $1-\delta$. Finally, Griffin recently showed [17] that similar results hold for Bloch eigenmodes (i.e., non-zero quasimomentum) for periodic point scatterers in three dimensions, provided a certain Diophantine condition on the aspect ratio holds.

1.3. Scarring and QUE for some other models. For quantum ergodic systems almost all eigenfunctions equidistribute, but in general not much is known about the (potential) subset of exceptional eigenfunctions giving non-uniform quantum limits. In some cases Quantum Unique Ergodicity is known to hold; notable examples are Hecke eigenfunctions on modular surfaces [28,41] and "quantized cat maps" [23,25]. For these models there exist large commuting families of "Hecke symmetries" that also commute with the quantized Hamiltonian, and it is then natural to consider joint eigenfunctions of the full family of commuting operators. Other examples arise when the underlying classical dynamics is uniquely ergodic, QUE is then "automatic", e.g., see [30,33].

On the other hand there are quantum ergodic systems exhibiting scarring. For example, if Hecke symmetries are not taken into account, quantized cat maps can have very large spectral degeneracies. Using this, Faure, Nonnenmacher and de-Bievre [13] proved that scars occur in this model. For higher dimensional analogues of cat maps, Kelmer found a scar construction not involving spectral degeneracies, but rather certain invariant rational isotropic subspaces [23,24].

We also note that Berkolaiko, Keating, and Winn have shown [4,5] that position scarring can occur for quantum star graphs. e.g., for certain star graphs with a fixed (but arbitrarily large) number of bonds, there exists quantum limits supported only on two bonds (consequently, if the graph is viewed as embedded in Euclidean space, there is also localization in momentum).

Another way to construct localized eigenfunctions is to use "bouncing ball quasimodes". For example, functions of the form $\psi_{n}(x, y)=f(x) \sin (n y)$ are approximate Laplace eigenfunctions on a stadium shaped domain (say with Dirichlet boundary conditions), and semiclassically localize on vertical periodic trajectories. Hassell showed [20] that for a generic aspect ratio stadium, there are few eigenvalues near $n^{2}$ and hence $\psi_{n}$ overlaps strongly with an eigenfunction $\phi_{n}$, with eigenvalue near $n^{2}$, which also localizes on vertical periodic trajectories.

In $[3,29,42]$ the asymptotics for the number of "bouncing ball eigenfunctions" having eigenvalue at most $E$ were studied for some ergodic billiards having parallell walls.

e.g., for the stadium billiard, numerics and an "adiabatic separation ansatz" indicated that the number of bouncing ball eigenfunctions, with eigenvalue at most $E$, is of order $E^{3 / 4}$. This is to be compared with the Weyl asymptotic $c \cdot E$, and hence bouncing ball eigenfunctions are fairly rare. Interestingly, in [3] it was argued that given any $\delta \in(1 / 2,1)$, there exists a Sinai type billiard, with the area of the internal obstacle bounded from below, whose bouncing ball eigenfunction count is of order $c_{\delta} \cdot E^{\delta}$. In a sense, Theorem 3 is a "shrinking obstacle" analog of this phenomena, as bouncing ball eigenfunction are highly localized in the momentum representation.

1.4. Outline of the proofs. The proofs are based on finding new eigenvalues $\lambda$ that are "abnormally close" to certain old eigenvalues that have "abnormally small" multiplicity. More precisely, after rewriting equation (6) as 


$$
\frac{r_{d}(m)}{m-\lambda}-\frac{m}{m^{2}+1}+H_{m}(\lambda)=0
$$

where

$$
H_{m}(\lambda):=\sum_{\substack{n \neq m \\ n \in \mathcal{N}_{d}}} r_{d}(n)\left(\frac{1}{n-\lambda}-\frac{n}{n^{2}+1}\right)-F(\lambda),
$$

we show that for certain $m \in \mathcal{N}_{d}$ there exists a new eigenvalue $\lambda$ such that $|m-\lambda| \ll$ $\sqrt{r_{d}(m) / H_{m}^{\prime}(m)}$. Using number theoretic techniques, we then find a subsequence of integers $m$ such that $\sqrt{r_{d}(m) / H_{m}^{\prime}(m)}$ is small and $r_{d}(m)$ is bounded, and thus get a control on the distance of a new eigenvalue from these $m$.

In spirit, we use a nearby old eigenvalue $m^{\prime}$, with $r_{d}\left(m^{\prime}\right)$ much larger than $r_{d}(m)$, to "push" the new eigenvalue $\lambda$ very close to $m$ (though it must be stressed that we do not know whether $\lambda>m$ or $\lambda<m)$. Having $\sqrt{r_{d}(m) / H_{m}^{\prime}(m)}$ bounded turns out to imply that significant $L^{2}$-mass of the Green's function $G_{\lambda}$ (cf. (2)) is carried by the finite sum

$$
\sum_{v \in \mathbb{Z}^{d}:|v|^{2}=m} \frac{\exp \left(-i v \cdot x_{0}\right)}{|v|^{2}-\lambda} e^{i v \cdot x}
$$

which, since $r_{d}(m)$ is bounded, gives momentum localization on $\left\{v /|v|: v \in \mathbb{Z}^{d}\right.$ : $|v|=m\} \subset \mathbb{S}^{d-1}$. To obtain non-uniform quantum limits for $d=2$, we impose the additional constraint that the set $\left\{v \in \mathbb{Z}^{2}:|v|^{2}=m\right\}$ contains two points $v, v^{\prime}$ such that $\left|v-v^{\prime}\right|$ is bounded; for $m=n^{2}+1$ we may take $v=(n, 1)$ and $v^{\prime}=(n,-1)$; using that $r_{2}(m)$ is bounded we then obtain positive mass on the Fourier mode $e^{i w \cdot x}$, where $w=v-v^{\prime}=(0,2)$. (In contrast, for $d=3$, the minimum distance between distinct lattice points $v_{i}, v_{i}^{\prime} \in \mathbb{Z}^{3}$ such that $\left|v_{i}\right|^{2}=\left|v_{i}^{\prime}\right|^{2}=m_{i}$ must grow if $m_{i} \rightarrow \infty$ in such a way that $r_{3}\left(m_{i}\right)$ remains bounded).

The paper is organized as follows: In Sect. 2 we set the necessary background for the point scatterer model, then give some number theoretic background, and in Sect. 3 we prove some auxiliary analytic and number theoretic results needed in the proofs of our main theorems. In Sects. 4 and 5 we prove Theorems 1 and 2, and Sect. 6 contains the proof of Theorem 3 .

\section{Background}

In this section we briefly review some results and definitions about point scatterers and give a short number theoretic background.

2.1. Point scatterers on the flat torus. We begin with the point scatterers, and recall the definition and properties of the quantization of observables (see [27,35] for more details; further background can be found in $[43,45])$.

2.1.1. Basic definitions and properties. For $d=2,3$ we consider the restriction of the Laplacian $-\Delta$ on

$$
D_{0}:=C_{0}^{\infty}\left(\mathbb{T}^{d} \backslash\left\{x_{0}\right\}\right) .
$$


The restriction is symmetric though not self-adjoint, but by von Neumann's theory of self adjoint extensions there exists a one-parameter family of self-adjoint extensions; for $\varphi \in(-\pi, \pi]$ there exists a self-adjoint extension $H_{\varphi}$, where the case $\varphi=\pi$ corresponds to the unperturbed Laplacian. The spectrum of $H_{\varphi}$ consists of two types of eigenvalues and eigenfunctions:

1. Eigenvalues of the unperturbed Laplacian, and the corresponding eigenfunctions that vanish at $x_{0}$. The multiplicities of the new eigenvalues are reduced by 1 , due to the constraint of vanishing at $x_{0}$.

2. New eigenvalues $\lambda \in \mathbb{R}$ satisfying the equation

$$
\sum_{n \in \mathcal{N}_{d}} r_{d}(n)\left(\frac{1}{n-\lambda}-\frac{n}{n^{2}+1}\right)=c_{0} \tan \left(\frac{\varphi}{2}\right) .
$$

For $\lambda \in \mathbb{R}$ satisfying (8), the corresponding Green's function

$$
\begin{aligned}
G_{\lambda}\left(x, x_{0}\right) & =(\Delta+\lambda)^{-1} \delta_{x_{0}} \\
& =-\frac{1}{4 \pi^{2}} \sum_{v \in \mathbb{Z}^{d}} \frac{\exp \left(-i v \cdot x_{0}\right)}{|v|^{2}-\lambda} e^{i v \cdot x}, \quad x \neq x_{0}
\end{aligned}
$$

is an eigenfunction, and

$$
g_{\lambda}\left(x, x_{0}\right):=\frac{G_{\lambda}\left(x, x_{0}\right)}{\left\|G_{\lambda}\right\|}=\frac{\sum_{v \in \mathbb{Z}^{d}} \frac{\exp \left(-i v \cdot x_{0}\right)}{|v|^{2}-\lambda} e^{i v \cdot x}}{\left(\sum_{n \in \mathcal{N}_{d}} \frac{r_{d}(n)}{|n-\lambda|^{2}}\right)^{1 / 2}}
$$

is an $L^{2}$-normalized eigenfunction.

2.1.2. Strong coupling. In the physics literature, for $d=2$, the "strong coupling limit" is considered more relevant than the weak coupling limit given by (8). A convenient way of arriving at this quantization is by truncating the summation in (8) outside an energy window of size $O\left(\lambda^{\eta}\right)$ for any fixed $\eta>131 / 146$ (cf. [43, Section 3]). This leads to the following spectral equation for the new eigenvalues:

$$
\sum_{\substack{n \in \mathcal{N}_{2} \\\left|n-n_{+}(\lambda)\right|<n_{+}(\lambda)^{\eta}}} r_{2}(n)\left(\frac{1}{n-\lambda}-\frac{n}{n^{2}+1}\right)=c_{0} \tan \left(\frac{\varphi}{2}\right) .
$$

2.1.3. Quantization of observables. Given a smooth observable $a(x, \xi)$ on $S^{*}\left(\mathbb{T}^{d}\right) \simeq$ $\mathbb{T}^{d} \times \mathbb{S}^{d-1}$ we define the quantization of it as a pseudo-differential operator $\operatorname{Op}(a)$ : $C^{\infty}\left(\mathbb{T}^{d}\right) \rightarrow C^{\infty}\left(\mathbb{T}^{d}\right)$. We refer the reader to [27] for details on the two dimensional case, and [45] for the three dimensional case. We are mainly focusing on either pure momentum, or pure position observables, that is

$a(x, \xi)=a(\xi) \in C^{\infty}\left(\mathbb{S}^{d-1}\right)$, or $a(x, \xi)=a(x) \in C^{\infty}\left(\mathbb{T}^{d}\right)$ respectively. Given $f(x) \in C^{\infty}\left(\mathbb{T}^{d}\right)$, the action of a pure position observable $a=a(x) \in C^{\infty}\left(\mathbb{T}^{d}\right)$ is given by

$$
(\mathrm{Op}(a) f)(x)=a(x) f(x),
$$


whereas the action of a pure momentum observable $a=a(\xi) \in C^{\infty}\left(\mathbb{S}^{d-1}\right)$ is given by

$$
(\mathrm{Op}(a) f)(x)=\sum_{v \in \mathbb{Z}^{d}} a\left(\frac{v}{|v|}\right) \widehat{f}(v) e^{i v \cdot x}
$$

in particular, for pure momentum observables we have

$$
\langle\mathrm{Op}(a) f, f\rangle=\sum_{v \in \mathbb{Z}^{d}} a\left(\frac{v}{|v|}\right)|\widehat{f}(v)|^{2} .
$$

(some care is required for the terms for which $v=0$; it is convenient to interpret $a(v /|v|$ ) for $v=0$ as the spherical average $\int_{\mathbb{S}^{d-1}} a(\xi) d \xi$, with the measure normalized so that $\left.\int_{\mathbb{S}^{d-1}} 1 d \xi=1\right)$.

2.2. Number theoretic background. We begin by recalling some convenient notations. We use the Landau symbols $O$ and $o$ and the Vinogradov symbols $\gg$ and $\ll$ with their usual meaning. That is, $A=O(B), A \ll B$ and $B \gg A$ are all equivalent to the fact that $|A|<c B$ holds for some absolute constant $c>0$, while $A=o(B)$ means that $A / B \rightarrow 0$. In case the implied constant is allowed to depend on some parameter (say $\epsilon)$, we write $A=O_{\epsilon}(B)$ or $A \ll_{\epsilon} B$.

2.2.1. Integers that are sums of 2 or 3 squares. We begin with a short summary about integers that can be represented as sums of $d$ squares for $d=2$ or 3 .

Sums of 2 squares: It is well known (e.g., see [11]) that $r_{2}(n)$ is determined by the prime factorization of $n$. If we write

$$
n=2^{a_{0}} p_{1}^{a_{1}} \ldots p_{r}^{a_{r}} q_{1}^{b_{1}} \ldots q_{l}^{b_{l}}
$$

where the $p_{i}$ 's are primes all $\equiv 1(\bmod 4)$, and the $q_{i}$ 's are primes all $\equiv 3(\bmod 4)$, then $n$ is a sum of two squares if and only if all the $b_{i}$ are even, and $r_{2}(n)=4 d\left(p_{1}^{a_{1}} \ldots p_{r}^{a_{r}}\right)$, where $d(m):=\sum_{l \mid m} 1$ is the divisor function.

Sums of 3 squares: For $d=3$, any number $n$ that is not of the form $n=4^{a} n_{1}$, where $4 \nmid n_{1}$ and $n_{1} \equiv 7(\bmod 8)$ can be represented as a sum of 3 squares. Moreover, $r_{3}(4 n)=r_{3}(n)$ for any $n \in \mathbb{Z}$, and if we let $R_{3}(n)$ denote the number of primitive representation of $n$ as a sum of 3 squares (that is the number of ways to write $n=x^{2}+y^{2}+z^{2}$ with $x, y, z$ coprime), we can relate $r_{3}(n)$ to class numbers of quadratic imaginary fields as follows (cf. [18, Theorem 4, p. 54]):

$$
r_{3}(n)=\sum_{d^{2} \mid n} R_{3}\left(\frac{n}{d^{2}}\right), \quad R_{3}(n)=\pi^{-1} G_{n} \sqrt{n} L\left(1, \chi_{n}\right),
$$

where

$$
G_{n}=\left\{\begin{array}{l}
0 \quad n \equiv 0,4,7 \quad(\bmod 8) \\
16 \quad n \equiv 3 \quad(\bmod 8) \\
24 \quad n \equiv 1,2,5,6 \quad(\bmod 8)
\end{array}\right.
$$


and

$$
L\left(s, \chi_{n}\right)=\sum_{m=1}^{\infty} \chi_{n}(m) m^{-s}
$$

is the Dirichlet $L$-series associated with the multiplicative character $\chi_{n}(m):=(-4 n / m)$, where $(-4 n / m)$ is the Kronecker symbol. By a celebrated theorem of Siegel, for any $\epsilon>0, L\left(1, \chi_{n}\right) \gg_{\epsilon} n^{-\epsilon}$ and thus, for $n \not \equiv 0,4,7 \bmod 8$,

$$
r_{3}(n) \geq R_{3}(n) \gg_{\epsilon} n^{1 / 2-\epsilon} \text {. }
$$

Further, given an integer $n$ that is a sum of 3 squares, let

$$
\Omega(n):=\left\{\frac{(x, y, z)}{\sqrt{n}}:(x, y, z) \in \mathbb{Z}^{3}, x^{2}+y^{2}+z^{2}=n\right\} \subset \mathbb{S}^{2} .
$$

Fomenko-Golubeva and Duke showed (see [9,16], or [10, Lemma 2]) that the sets $\Omega(n)$ equidistribute in $\mathbb{S}^{2}$ as $r_{3}(n) \rightarrow \infty$ (or equivalently $n_{1} \rightarrow \infty$ ) inside $\mathcal{N}_{3}$. Namely, there exists $\alpha>0$, such that for any spherical harmonic $Y(x)$, there is significant cancellation in the following analogue of a Weyl sum

$$
W_{Y}(n):=\sum_{\xi \in \Omega(n)} Y(\xi)
$$

in the sense that

$$
W_{Y}(n) \ll n_{1}^{1 / 2-\alpha} \ll n^{1 / 2-\alpha}
$$

where the implied constant is independent of $n$.

2.3. Sieve method results. In order to prove Theorem 3 and the second part of Theorem 2 , we will need some sieve results that show the existence of various infinite sequences of integers with bounded number of prime divisors. We first recall a few definitions. A positive integer $n$ is called $r$-almost prime if $n$ has at most $r$ prime divisors. We denote by $P_{r}$ the set of all $r$-almost prime integers. A finite set of polynomials $\mathcal{F}=$ $\left\{F_{1}(x), \ldots, F_{k}(x)\right\} \subset \mathbb{Z}[x]$ is called admissible if $F(x):=\prod_{i=1}^{k} F_{i}(x)$ has no fixed prime divisors, that is the equation $F(x) \equiv 0(\bmod p)$ has less than $p$ solutions for any prime $p$. The following theorem combines results from $[15,19,31]$ :

Theorem 5. Let $F_{1}(x), \ldots, F_{k}(x) \in \mathbb{Z}[x](k \geq 1)$ be a finite admissible set of irreducible polynomials, and let $F(x):=F_{1}(x) \cdots F_{k}(x)$. Let $G$ denote the degree of $F$. Then,

1. [19, Theorem 10.4] There exists an integer $R(k, G)$, such that for any $r>R(k, G)$, as $x \rightarrow \infty$,

$$
\#\left\{n \in \mathbb{Z}, n \leq x: F(n) \in P_{r}\right\} \gg \frac{x}{\log ^{k} x}
$$

2. [15, Theorem 25.4] If $k=1$, then one can take $R(k, G)=G+1$, and therefore as $x \rightarrow \infty$

$$
\#\left\{n \leq x: F(n) \in P_{G+1}\right\} \gg \frac{x}{\log x}
$$


3. [31, Theorem 3.4] If $k$ is large enough, and $F_{1}(x), \ldots, F_{k}(x)$ are all linear with positive coefficients, then as $x \rightarrow \infty$

$$
\mid\left\{n \leq x: \text { at least two of the } F_{i}(n), 1 \leq i \leq k \text {, are prime }\right\} \mid \gg \frac{x}{(\log x)^{k}}
$$

\section{Auxiliary Results}

Before proceeding to the proofs of the main theorems, we begin with a few auxiliary results. For the benefit of the reader we note that Lemma 6 is relevant for all theorems, Lemma 7 is used in the proof of Theorem 2, whereas Lemma 8 and Proposition 9 are used in the proof of Theorem 3.

3.1. Nearby zeros. The following simple result will be crucial in finding integers $m$ in the old spectrum for which there exist a nearby new eigenvalue $\lambda$.

Lemma 6. Let I be a closed symmetric interval containing zero, and let $f$ be $C^{1}$ function on I. Let $A>0$ be a real number, and assume that $B:=\min _{\delta \in I} f^{\prime}(\delta)>0$. If $\sqrt{A / B} \in I$ there exists $\delta_{0} \in[-\sqrt{A / B}, \sqrt{A / B}]$ such that

$$
f\left(\delta_{0}\right)=A / \delta_{0}
$$

Proof. Let $I^{+}=I \cap[0, \infty]$, and let $I^{-}=I \cap[-\infty, 0]$. For $\delta \in I^{+}$, we have $f(\delta) \geq$ $f(0)+B \delta$. Similarly, for $\delta \in I^{-}, f(\delta) \leq f(0)+B \delta$. Thus, since $A, B>0$, if

$$
f(0)+B \delta_{1}=A / \delta_{1}
$$

for $\delta_{1} \in I^{+}$, there exists $\delta_{0} \in\left[0, \delta_{1}\right]$ such that $f\left(\delta_{0}\right)=A / \delta_{0}$. Similarly, if $f(0)+B \delta_{1}=$ $A / \delta_{1}$ for $\delta_{1} \in I^{-}$, there exists $\delta_{0} \in\left[\delta_{1}, 0\right]$ such that $f\left(\delta_{0}\right)=A / \delta_{0}$.

To conclude the proof it is enough to show that

$$
f(0)+B \delta=A / \delta
$$

has a solution in $[-\sqrt{A / B}, \sqrt{A / B}]$, but this is clear since $B \delta^{2}+f(0) \delta-A=0$ has at least one root $\delta_{1}$ for which $\left|\delta_{1}\right| \leq \sqrt{A / B}$.

3.2. Sequences of sums of two squares. In this section we will show the existence of "good sequences" of integers $m=n^{2}+1$ such that $r_{2}(m)$ is bounded, while $r_{2}(m+3)$ is "large". For such $m$ we can then find a new eigenvalue $\lambda$ such that $|\lambda-m|$ is small, and $g_{\lambda}$ is localized in both momentum and position.

Lemma 7. Given $\gamma \in(0,1 / 10)$ there exists an infinite set $\mathcal{M}_{\gamma} \subset \mathcal{N}_{2}$ with the following properties: $\forall m \in \mathcal{M}_{\gamma}$

$$
m=n^{2}+1
$$

for some $n \in \mathbb{Z}^{+}$,

$$
r_{2}(m) \leq 32
$$

and

$$
r_{2}(m+3) \geq 10 r_{2}(m) / \gamma^{2}
$$


Proof. We apply part (2) of Theorem 5 in the following setting: For $K \in \mathbb{Z}^{+}$define

$$
P(K)=\prod_{p \equiv 1} p,
$$

and $r(K) \in \mathbb{Z}^{+}$solving the following congruences:

$$
\begin{aligned}
& r(K)^{2}+4 \equiv 0 \quad(\bmod p) \text { if } p \equiv 1 \quad(\bmod 4), p \leq K \\
& r(K) \equiv 0 \quad(\bmod 2)
\end{aligned}
$$

Note that the latter equation has a solution by the Chinese remainder theorem together with -4 being a quadratic residue for any prime $p \equiv 1(\bmod 4)$. We may take $0 \leq$ $r(K)<2 P(K)$ but any fixed choice will suffice. Let $f(x)=x^{2}+1$, and let $x_{K}(n)=$ $2 P(K) \cdot n+r(K)$. The polynomial $F(n):=f\left(x_{K}(n)\right)$ satisfies all the conditions of the theorem (it is irreducible and no prime divides all coefficients), and therefore there are infinitely many $n$ such that $F(n)$ has at most 3 prime factors, and in particular $r_{2}(F(n)) \leq 32$. By construction, $F(n)+3=x_{k}(n)^{2}+2^{2} \equiv 0(\bmod p)$ for $p \leq K$ and $p \equiv 1(\bmod 4)$, hence $r_{2}(F(n)+3) \geq 4 \cdot 2^{\pi(K ; 1,4)}$, where $\pi(K ; 1,4)$ is the number of primes occurring in the product defining $P(K)$. By choosing $K$ appropriately, we get that

$$
r_{2}(F(n)+3) \geq 4 d(P(K)) \geq 2^{\pi(K ; 1,4)+2} \geq 320 / \gamma^{2} \geq 10 r_{2}(F(n)) / \gamma^{2}
$$

Lemma 8. Given $H, R \geq 2$ there exists elements $0<a_{1}<a_{2} \ldots<a_{H}$ in $\mathcal{N}_{2}$ such that $a_{H}-a_{1}<H^{2}, 0<r_{2}\left(a_{1}\right)<\ldots<r_{2}\left(a_{H}\right) \ll_{H} R^{H}$, and

$$
r_{2}\left(a_{i+1}\right)>R \cdot r_{2}\left(a_{i}\right)
$$

holds for some $0<i<H$.

Proof. Define

$$
Q_{1}=Q_{1}(H):=\prod_{p<2 H} p^{E_{p}}
$$

where the exponents $E_{p}$ are chosen as follows: let $E_{p}=1$ if $p \equiv 3 \bmod 4$, otherwise let $E_{p}$ be the minimal integer so that $p^{E_{p}}>H^{2}$.

Further, let $q_{1}<q_{2}<\cdots<q_{H}$ be primes congruent to $1 \bmod 4$, chosen so that $q_{1}>2 H$, and given integer exponents $e_{1}, \ldots, e_{H} \geq 1$, define

$$
Q_{2}=Q_{2}\left(e_{1}, e_{2}, \ldots, e_{H}\right):=\prod_{i \leq H} q_{i}^{e_{i}}
$$

and finally let $Q:=Q_{1} \cdot Q_{2}$.

By the Chinese remainder theorem we may find $\gamma \bmod Q$ such that the following holds:

$$
\begin{aligned}
& \gamma \equiv 0 \quad \bmod p^{E_{p}} \quad \text { if } p \equiv 1,2 \quad \bmod 4 \text { and } p<2 H \\
& \gamma \equiv 1 \quad \bmod p \quad \text { if } p \equiv 3 \quad \bmod 4 \text { and } p<2 H
\end{aligned}
$$

and for each prime $q_{i} \mid Q_{2}$ so that

$$
q_{i}^{e_{i}} \|\left(\gamma^{2}+i^{2}\right)
$$


Letting $d_{i}=\left(Q_{1}^{2}, \gamma^{2}+i^{2}\right)$ we define polynomials $G_{i} \in \mathbb{Q}[t]$ by

$$
G_{i}(t):=\left((Q t+\gamma)^{2}+i^{2}\right) /\left(q_{i}^{e_{i}} d_{i}\right), \quad i=1,2, \ldots, H .
$$

By definition, $d_{i} \mid \gamma^{2}+i^{2}$, and (22) implies that $q_{i}^{e_{i}} \mid \gamma^{2}+i^{2}$. Thus, since $\left(Q_{1}, Q_{2}\right)=1$ implies that $\left(q_{i}, d_{i}\right)=1$, we find that $q_{i}^{e_{i}} d_{i} \mid \gamma^{2}+i^{2}$ and consequently $G_{i}(t) \in \mathbb{Z}[t]$ for all $i$.

Claim. $\left\{G_{i}\right\}_{i=1}^{H}$ is an admissible set of polynomials (i.e., $\prod_{i=1}^{H} G_{i}(x)$ does not have any fixed prime divisors).

To prove the claim we argue as follows: If $p>2 H$ and all $G_{i}$ are nonconstant modulo $p$ (i.e., $p \nmid Q$ ) there are at most $2 H$ residues $n(\operatorname{modulo} p)$ for which $G_{i}(n) \equiv 0 \bmod p$ for some $i$. Hence there exist $n \in \mathbb{Z}$ such that $\prod_{i=1}^{H} G_{i}(n) \not \equiv 0 \bmod p$.

On the other hand, if $p>2 H$ and $p \mid Q$ then $p=q_{i}$ for some $i$, and by the definition of $G_{i}$ [in particular, recall (22)], we find that $G_{i}(n) \not \equiv 0 \bmod q_{i}$ for all $n \in \mathbb{Z}$. Moreover, if $j \neq i$,

$$
\gamma^{2}+j^{2} \equiv \gamma^{2}+i^{2}+j^{2}-i^{2} \equiv j^{2}-i^{2} \not \equiv 0 \quad \bmod q_{i}
$$

(as $0<|i-j|<H, 0<i+j<2 H$ and $q_{i}>2 H$ ), and thus $G_{j}(n) \not \equiv 0 \bmod q_{i}$ for all $n \in \mathbb{Z}$.

For $p<2 H$ we argue as follows: if $p \equiv 3 \bmod 4,(21)$ gives that $\gamma^{2}+i^{2} \not \equiv 0$ $\bmod p$ for all $i \in \mathbb{Z}$. Otherwise, $i^{2} \leq H^{2}<p^{E_{p}}$ by our choice of $E_{p}$, and since $\gamma$ was chosen so that $\gamma \equiv 0 \bmod p^{E_{p}}$ [recall (20)], we find that $\gamma^{2}+i^{2} \equiv i^{2} \not \equiv 0 \bmod p^{2 E_{p}}$, as $i^{2} \leq H^{2}$ and $p^{E_{p}}>H^{2}$. Consequently $\left(\gamma^{2}+i^{2}\right) / d_{i}$ is not divisible by $p$. The proof of the claim is concluded.

Now, given an integer $r>0$, let $P_{r}$ denote the set of integers that can be written as a product of at most $r$ primes, as in Sect. 2.3. Since the polynomials $\left\{G_{i}(x)\right\}_{i=1}^{H}$ form an admissible set, part (1) of Theorem 5 implies that there exists some $r>0$ (only depending on $H$ ) such that

$$
\prod_{i=1}^{H} G_{i}(n) \in P_{r}
$$

for infinitely many $n$. Given such an $n$, let $m_{i}=G_{i}(n)$; then each $m_{i}$ is a sum of squares that in addition has at most $r$ prime factors. Consequently, if we set $a_{i}=m_{i} \cdot q_{i}^{e_{i}} \cdot d_{i}$, we find that $a_{i} \in \mathcal{N}_{2}$ for all $1 \leq i \leq H$, and that

$$
e_{i}+1 \leq r_{2}\left(a_{i}\right) \leq 4 C \cdot\left(e_{i}+1\right)
$$

where $C=C(H) \geq 1$ is independent of the exponents $e_{1}, \ldots, e_{H}$. Choosing $e_{1}, \ldots, e_{H}$ appropriately we can ensure that $r_{2}\left(a_{i+1}\right)>R \cdot r_{2}\left(a_{i}\right)$ holds for all $i$, as well as that $r_{2}\left(a_{H}\right) \ll_{H} C^{H} R^{H} \ll_{H} R^{H}$ (a somewhat better $C$-dependency can be obtained but we shall not need it).

Finally, since

$$
a_{i}=m_{i} q_{i}^{e_{i}} d_{i}=G_{i}(n) q_{i}^{e_{i}} d_{i}=(Q n+\gamma)^{2}+i^{2}
$$

we find that $a_{H}-a_{1}=H^{2}-1<H^{2}$ and the proof of Lemma 8 is concluded. 
The following proposition might be of independent interest-using the full power of [31], the method of the proof in fact gives the following: given $k \geq 2$ and $R>1$ there exists $A>0$ such that, as $x \rightarrow \infty$, there are $\gg x /(\log x)^{A}$ integers $n \leq x$ such that $r_{2}\left(n+h_{i+1}\right) \geq R r_{2}\left(n+h_{i}\right)$ holds for $i=1, \ldots, k-1$ and $0<h_{1}<h_{2}<\cdots<h_{k} \ll_{k} 1$. For simplicity we only state and prove it for $k=2$.

Proposition 9. There exist an integer $H \geq 1$ with the following property: for all sufficiently large $R$ there exist an integer $h \in\left(0, H^{2}\right)$ such that

$$
\left|\left\{n \in \mathcal{N}_{2}: n \leq x, 0<r_{2}(n) \ll R^{H}, r_{2}(n+h) \geq R \cdot r_{2}(n)\right\}\right| \gg_{R} x /(\log x)^{H}
$$

as $x \rightarrow \infty$.

Proof. By part (3) of Theorem 5 there exists integers $i, j$ such that $0<i<j \leq H$ with the property that

$$
\mid\left\{n \leq x: F_{i}(n), F_{j}(n) \text { both prime }\right\} \mid \gg x / \log ^{H} x
$$

for $\left\{F_{1}, F_{2}, \ldots, F_{H}\right\}$ any admissible set of $H$ linear forms, provided $H$ is sufficiently large. For such an $H$, and a given (large) $R$, Lemma 8 shows there exists $a_{1}, \ldots, a_{H}>0$ such that

$$
r_{2}\left(a_{i+1}\right) \geq R \cdot r_{2}\left(a_{i}\right)>0
$$

for $1 \leq i<H$, and $r_{2}\left(a_{H}\right) \ll R^{H}$. If we define

$$
F_{i}(n):=a_{i} \cdot n+1
$$

for $1 \leq i \leq H$ we obtain a set of $H$ admissible linear forms (here admissibility is trivial since $F_{i}(0) \not \equiv 0 \bmod p$ for any prime $p$ ), hence there exists $i, j$ with $j>i$ such that

$$
\mid\left\{n \leq x: F_{i}(n), F_{j}(n) \text { both prime }\right\} \mid \gg x / \log ^{H} x
$$

Further, given primes $p=F_{i}(n)$ and $p^{\prime}=F_{j}(n)$, define $m=a_{j} \cdot p$ and $m^{\prime}=a_{i} \cdot p^{\prime}$. Now, since $a_{i} \equiv 0 \bmod 4$ for all $i, F_{i}(n) \equiv 1 \bmod 4$ for all $n$, hence $p, p^{\prime} \equiv 1 \bmod 4$ and consequently $m, m^{\prime} \in \mathcal{N}_{2}$. Further, $m^{\prime} \ll_{R} x$; letting $h=m-m^{\prime}$ we find that

$$
h=m-m^{\prime}=a_{j} \cdot F_{i}(n)-a_{i} \cdot F_{j}(n)=a_{j}-a_{i}
$$

and thus $0<h<H^{2}$. Moreover,

$$
r_{2}(m)=r_{2}\left(p \cdot a_{j}\right)=2 \cdot r_{2}\left(a_{j}\right)
$$

and similary $r_{2}\left(m^{\prime}\right)=2 \cdot r_{2}\left(a_{i}\right)$. Since $r_{2}\left(a_{j}\right) \geq R \cdot r_{2}\left(a_{i}\right)$ we find that

$$
r_{2}\left(m^{\prime}\right) \geq R \cdot r_{2}(m)
$$

and that $r_{2}(m)=2 \cdot r_{2}\left(a_{i}\right) \ll R^{H}$. Taking $n=m^{\prime}$ and $h=m-m^{\prime}$ we find that the number of $n \ll_{R} x$ with the desired property is $\gg x /(\log x)^{H}$, thus concluding the proof. 


\section{Proof of Theorem 1}

By a standard approximation argument it is enough to prove the statement for some orthogonal basis of smooth observables (e.g., see [27, Section 4]). Thus, given $m, l$ let $Y=Y_{l, m}(\xi)$ denote a (say $L^{2}$-normalized) spherical harmonic of degree $l$ and order $m$ on $\mathbb{S}^{2}$. Further, given $Y$ and $v \in \mathbb{Z}^{3}$, define

$$
e_{v, Y}(x, \xi):=e^{i v \cdot x} \cdot Y(\xi)
$$

Since $\mu_{\Omega(l)}\left(e_{w, Y}\right)=0$ for $w \neq 0$, we begin by showing that the same holds for any semiclassical measure. The following result is implicit in the proof of [45, Proposition 7.1]; it is stated only for a limit along a full density subset of new eigenvalues, but the proof gives a slightly stronger result.

Lemma 10. If $w \in \mathbb{Z}^{3}$ is nonzero, then

$$
\lim _{\lambda \rightarrow \infty, \lambda \notin \mathcal{N}_{3}}\left\langle\mathrm{Op}\left(e_{w, Y}\right) g_{\lambda}, g_{\lambda}\right\rangle=0
$$

Proof. For the convenience of the reader, we briefly recall Yesha's argument. Given $\delta \in(0,1 / 28)$ let $L:=\lambda^{\delta}$ and define a truncated Green's function

$$
G_{\lambda, L}\left(x, x_{0}\right):=\sum_{v \in \mathbb{Z}^{3}:|v|^{2}-\lambda \mid<L} \frac{e^{i v \cdot\left(x-x_{0}\right)}}{|v|^{2}-\lambda},
$$

and let $g_{\lambda, L}:=\frac{G_{\lambda, L}}{\left\|G_{\lambda, L}\right\|_{2}}$ denote the corresponding $L^{2}$ normalized function. Then (cf. [45, Section 4]) $\left\|g_{\lambda, L}-g_{\lambda}\right\|_{2} \rightarrow 0$ as $\lambda \rightarrow \infty$, and for all smooth observables $f \in$ $C^{\infty}\left(S^{*}\left(\mathbb{T}^{3}\right)\right)$,

$$
\left|\left\langle\mathrm{Op}(f) g_{\lambda}, g_{\lambda}\right\rangle-\left\langle\mathrm{Op}(f) g_{\lambda, L}, g_{\lambda, L}\right\rangle\right| \ll_{f}\left\|g_{\lambda}-g_{\lambda, L}\right\|_{2}^{2} .
$$

By [45, Proposition 7.1] (note that $w \neq 0$ ), $\left\langle\mathrm{Op}\left(e_{w, Y}\right) g_{\lambda, L}, g_{\lambda, L}\right\rangle \rightarrow 0$ as $\lambda \rightarrow \infty$, and hence $\left\langle\mathrm{Op}\left(e_{w, Y}\right) g_{\lambda}, g_{\lambda}\right\rangle \rightarrow 0$ as $\lambda \rightarrow \infty$ in such a way that $\lambda \notin \mathcal{N}_{3}$.

We next treat the case of pure momentum observables, i.e., $f \in C^{\infty}\left(S^{*}\left(\mathbb{T}^{3}\right)\right)$ of the form $f(x, \xi)=Y(\xi)$ for $Y(x)$ a spherical harmonic on $\mathbb{S}^{2}$. By the definition of $g_{\lambda}=\frac{G_{\lambda}}{\left\|G_{\lambda}\right\|}$ (cf. (10)), and the action of $\mathrm{Op}(Y)$ (cf. (14)) we get

$$
\begin{aligned}
\left\langle\mathrm{Op}(Y) g_{\lambda}, g_{\lambda}\right\rangle= & \frac{\sum_{v \in \mathbb{Z}^{3}} Y\left(\frac{v}{|v|}\right)\left(\frac{1}{|v|^{2}-\lambda}\right)^{2}}{\sum_{v \in \mathbb{Z}^{3}}\left(\frac{1}{|v|^{2}-\lambda}\right)^{2}}=\frac{\sum_{n \in \mathcal{N}_{3}} W_{Y}(n)\left(\frac{1}{n-\lambda}\right)^{2}}{\sum_{n \in \mathcal{N}_{3}} r_{3}(n)\left(\frac{1}{n-\lambda}\right)^{2}} \\
& =\frac{W_{Y}(m)+(m-\lambda)^{2} \sum_{n \in \mathcal{N}_{3} \backslash\{m\}} W_{Y}(n)\left(\frac{1}{n-\lambda}\right)^{2}}{r_{3}(m)+(m-\lambda)^{2} \sum_{n \in \mathcal{N}_{3} \backslash\{m\}} r_{3}(n)\left(\frac{1}{n-\lambda}\right)^{2}}
\end{aligned}
$$

for any $m \in \mathcal{N}_{3}$. Now, for $m \in \mathcal{N}_{3}$, define

$$
H_{m}(\lambda):=\sum_{n \in \mathcal{N}_{3} \backslash\{m\}} r_{3}(n)\left(\frac{1}{n-\lambda}-\frac{n}{n^{2}+1}\right)
$$


and rewrite the "new" eigenvalue equation (8) as

$$
\frac{r_{3}(m)}{m-\lambda}-\frac{m}{m^{2}+1}+H_{m}(\lambda)=c_{0} \tan \left(\frac{\varphi}{2}\right) \text {. }
$$

We can now apply Lemma 6 . Setting $\lambda=m+\delta$, let

$$
f_{m}(\delta):=H_{m}(m+\delta)-\frac{m}{m^{2}+1}-c_{0} \tan \left(\frac{\varphi}{2}\right) .
$$

Then

$$
f_{m}^{\prime}(\delta)=\sum_{n \in \mathcal{N}_{3} \backslash\{m\}} \frac{r_{3}(n)}{(n-m-\delta)^{2}}>0
$$

Notice that for $|\delta|<\frac{1}{2}$ there exists an absolute constant $C>1$ such that

$$
\frac{1}{C} f_{m}^{\prime}(0) \leq f_{m}^{\prime}(\delta) \leq C f_{m}^{\prime}(0)
$$

Equation (24) can now be rewritten as

$$
f_{m}(\delta)=\frac{r_{3}(m)}{\delta}
$$

hence, provided that we can find $m$ for which the bound $\sqrt{C r_{3}(m) / f_{m}^{\prime}(0)}<\frac{1}{2}$ holds, we may take $I=[-1 / 2,1 / 2]$ in Lemma 6 and obtain an eigenvalue $\lambda$ such that

$$
|\lambda-m|<\sqrt{\mathrm{Cr}_{3}(m) / f_{m}^{\prime}(0)} .
$$

To find $m$ for which the above bound is valid, we proceed as follows. For $l \in \mathcal{N}_{3}$ fixed, define

$$
\Omega(l):=\left\{\frac{v}{\|v\|}:\|v\|^{2}=l, v \in \mathbb{Z}^{3}\right\}
$$

and let $\mathcal{M}_{l}:=\left\{4^{k} l: k \in \mathbb{N}\right\}$. For $m \in \mathcal{M}_{l}$ we then have $r_{3}(m)=r_{3}(l)$, hence $r_{3}(m)$ is uniformly bounded; we also note that $\Omega(m)=\Omega(l)$. Since for any integer $m$ there exists an integer $m^{\prime} \not \equiv 0,4,7(\bmod 8)$ of bounded distance from $m$, (16) implies that

$$
f_{m}^{\prime}(0)=\sum_{n \in \mathcal{N}_{3}} \frac{r_{3}(n)}{|n-m|^{2}} \geq \frac{r_{3}\left(m^{\prime}\right)}{\left|m^{\prime}-m\right|^{2}} \gg r_{3}\left(m^{\prime}\right) \gg\left(m^{\prime}\right)^{1 / 2-\varepsilon} \gg m^{1 / 2-\varepsilon} \text {. }
$$

Since $r_{3}(m)$ is uniformly bounded for $m \in \mathcal{M}_{l}$, we find that

$$
\sqrt{C r_{3}(m) / f_{m}^{\prime}(0)}<m^{-1 / 4+\epsilon}
$$

for all sufficiently large $m \in \mathcal{M}_{l}$. By the above argument, we have thus found infinitely many $m$ for which there exist a nearby new eigenvalue $\lambda=\lambda_{m}$ satisfying $|m-\lambda|<$ $\sqrt{\mathrm{Cr}_{3}(l) / f_{m}^{\prime}(0)}<m^{-1 / 4+\epsilon}$. In fact, using (28) we can apply Lemma 6 again, to get that (26) holds for $C=1+O\left(m^{-1 / 4+\epsilon}\right)$ and $\delta=O\left(m^{-1 / 4+\epsilon}\right)$. Let $\tilde{\Lambda}_{l}$ be the sequence of 
these eigenvalues; for $\lambda \in \tilde{\Lambda}_{l}$ we then find, upon recalling the equality in (25), and that (26) is valid since $|m-\lambda|=O\left(m^{-1 / 4+\epsilon}\right)$, that

$$
\begin{aligned}
\mid m & -\left.\lambda\right|^{2} \sum_{n \in \mathcal{N}_{3} \backslash\{m\}} \frac{r_{3}(n)}{|n-\lambda|^{2}} \\
& \leq \frac{\left(1+o\left(m^{-1 / 4}\right)\right) r_{3}(l)}{f_{m}^{\prime}(0)} \sum_{n \in \mathcal{N}_{3} \backslash\{m\}} \frac{r_{3}(n)}{|n-\lambda|^{2}}=\left(1+O\left(m^{-1 / 4+\epsilon}\right)\right)^{2} r_{3}(l)
\end{aligned}
$$

which is bounded. We now define $\Lambda_{l}$ by chosing an infinite subset of $\tilde{\Lambda}_{l}$ such that the limit

$$
A_{l}:=\lim _{\lambda \in \Lambda_{l}} \frac{|m-\lambda|^{2}}{r_{3}(l)} \sum_{n \in \mathcal{N}_{3} \backslash\{m\}} \frac{r_{3}(n)}{|n-\lambda|^{2}} \geq 0
$$

exists; note that $A_{l} \leq 1$ by (29). Furthermore, for any spherical harmonic $Y$,

$$
|m-\lambda|^{2} \sum_{n \in \mathcal{N}_{3} \backslash\{m\}} \frac{\left|W_{Y}(n)\right|}{|n-\lambda|^{2}} \leq C r_{3}(l) / f_{m}^{\prime}(0) \sum_{n \in \mathcal{N}_{3} \backslash\{m\}} \frac{\left|W_{Y}(n)\right|}{|n-\lambda|^{2}} .
$$

We claim that the RHS converges to 0 as $m \rightarrow \infty$. To see this, write

$$
\sum_{n \in \mathcal{N}_{3} \backslash\{m\}} \frac{\left|W_{Y}(n)\right|}{|n-\lambda|^{2}}=\sum_{\substack{n \in \mathcal{N}_{3} \backslash\{m\} \\ n \leq m+m^{1 / 3}}} \frac{\left|W_{Y}(n)\right|}{|n-\lambda|^{2}}+\sum_{\substack{n \in \mathcal{N}_{3} \backslash\{m\} \\ n>m+m^{1 / 3}}} \frac{\left|W_{Y}(n)\right|}{|n-\lambda|^{2}} .
$$

For the first sum, using that $|\lambda-n|>1 / 2$ for $n \neq m$ together with the bound $W_{Y}(n) \ll$ $m^{1 / 2-\alpha}$ (using (17)) we find that for all $n \leq m+m^{1 / 3}$ in the summand, the first sum is $\ll m^{1 / 2-\alpha}$. For the second sum, the mean value theorem gives that

$$
W_{Y}(n) \ll n^{1 / 2-\alpha}=(n-m)^{1 / 2-\alpha}+O\left(\frac{m}{(n-m)^{1 / 2+\alpha}}\right)
$$

and thus,

$$
\begin{aligned}
\sum_{n>m+m^{1 / 3}} \frac{W_{Y}(n)}{|n-m|^{2}} & \ll \sum_{n-m>m^{1 / 3}} \frac{1}{|n-m|^{3 / 2+\alpha}}+O\left(\sum_{n-m>m^{1 / 3}} \frac{m}{|n-m|^{5 / 2+\alpha}}\right) \\
& \ll m^{-1 / 3(1 / 2+\alpha)}+m^{1-1 / 3(3 / 2+\alpha)} \ll m^{1 / 2-\alpha / 3} .
\end{aligned}
$$

Hence, since $f_{m}^{\prime}(0) \gg m^{1 / 2-\varepsilon}$ (cf. (28)),

$$
\frac{C r_{3}(l)}{f^{\prime}(0)} \sum_{n \in \mathcal{N}_{3}} \frac{W_{Y}(n)}{|n-m|^{2}} \ll \frac{m^{1 / 2-\alpha / 3}}{m^{1 / 2-\varepsilon}} \ll m^{-\alpha / 3+\varepsilon}
$$

Thus, for any fixed spherical harmonic $Y$, for $\lambda \in \Lambda_{l}$ tending to infinity, we have

$$
\left\langle\mathrm{Op}(Y) g_{\lambda}, g_{\lambda}\right\rangle= \begin{cases}\frac{W_{Y}(l)+o(1)}{\left(1+A_{l}+o(1)\right) r_{3}(l)}+O\left(\lambda^{-\alpha+\varepsilon}\right) & \text { if } Y \text { is non trivial } \\ 1 & \text { if } \mathrm{Y} \text { is trivial. }\end{cases}
$$

As $\lambda$ grows, these are the spherical harmonics coefficients of the measure $\frac{1}{1+A_{l}} \delta_{\Omega(l)}+$ $\frac{A_{l}}{1+A_{l}} v$, and the proof is concluded (recall that $v$ denotes the uniform measure, and that $A_{l} \leq 1$, hence the singular part has mass at least $1 / 2$ ). 


\section{Proof of Theorem 2}

We start by finding a sequence of new eigenvalues lying close to the set of old eigenvalues. To do so we will again use Lemma 6. Recall that

$$
F(\lambda)= \begin{cases}\text { Constant } & \text { (weak coupling) } \\ \left.\sum_{\substack{n \in \mathcal{N}_{2} \\\left|n-n_{+}(\lambda)\right| \geq n_{+}(\lambda)^{\eta}}} r_{n-\lambda}-\frac{1}{n^{2}+1}\right) & \text { (strong coupling) }\end{cases}
$$

and in analogy with the three dimensional case we define

$$
\begin{aligned}
H_{m}(\lambda) & =\sum_{n \in \mathcal{N}_{2} \backslash\{m\}} r_{2}(n)\left(\frac{1}{n-\lambda}-\frac{n}{n^{2}+1}\right)-F(\lambda) \\
& =\sum_{n \in I(\lambda) \backslash\{m\}} r_{2}(n)\left(\frac{1}{n-\lambda}-\frac{n}{n^{2}+1}\right)
\end{aligned}
$$

where (for some fixed $\eta>131 / 146$ )

$$
I(\lambda):= \begin{cases}\mathcal{N}_{2} \cap\left[n_{+}(\lambda)-n_{+}(\lambda)^{\eta}, n_{+}(\lambda)+n_{+}(\lambda)^{\eta}\right] & \text { (strong coupling) } \\ \mathcal{N}_{2} & \text { (weak coupling) }\end{cases}
$$

(for simplicity, we only treat the case $C=0$ in the weak coupling limit; the same argument works for any fixed $C$ ).

Proposition 11. Let $F(\lambda)$ be as above, and given $\gamma \in(0,1 / 10)$ let $\mathcal{M}_{\gamma}$ be the set of integers given by Lemma 7. Then, for any $m \in \mathcal{M}_{\gamma}$, there exists a new eigenvalue $\lambda$ such that $|\lambda-m| \leq \gamma$ and

$$
H_{m}^{\prime}(\lambda) \leq(1+O(\gamma)) \cdot \frac{r_{2}(m)}{(m-\lambda)^{2}}
$$

as $\gamma \rightarrow 0$.

Proof. Given $m \in M_{\gamma}$ we start by finding (at least one) nearby new eigenvalue. To do so, rewrite the eigenvalue equation [i.e., (8) in the weak coupling limit, or (11) in the strong coupling limit]

$$
\sum_{n \in S} r_{2}(n)\left(\frac{1}{n-\lambda}-\frac{n}{n^{2}+1}\right)=F(\lambda)
$$

as

$$
r_{2}(m)\left(\frac{1}{m-\lambda}-\frac{m}{m^{2}+1}\right)+H_{m}(\lambda)=0 .
$$

Thus, with $\lambda=m+\delta$, and defining $f(\delta)=H_{m}(m+\delta)-r_{2}(m) \frac{m}{m^{2}+1}$, we wish to find (small) solutions to

$$
f(\delta)=\frac{r_{2}(m)}{\delta}
$$


Now, by (33), $f^{\prime}(\delta)$ is always a sum of positive terms, hence we may drop all terms but one, say the one corresponding to $k=m+3$ (recall that $m=n^{2}+1$, hence $k=n^{2}+4$ is a sum of two squares), and find that

$$
f^{\prime}(\delta) \geq \frac{r_{2}(m+3)}{((m+3)-(m+\delta))^{2}}=\frac{r_{2}(m+3)}{(3-\delta)^{2}} \geq \frac{r_{2}(m+3)}{10}
$$

for $|\delta| \leq 1 / 10$. By Lemma 6 , there exists $\delta_{0}$ such that

$$
f\left(\delta_{0}\right)=\frac{r_{2}(m)}{\delta_{0}}
$$

and

$$
\left|\delta_{0}\right| \leq \sqrt{10 r_{2}(m) / r_{2}(m+3)} \leq \gamma .
$$

Using the above estimate on $\delta$ we next show that the lower bound on $f^{\prime}(\delta)$ is essentially given by the size of $H_{m}^{\prime}(m)$. With $m_{-}, m_{+} \in \mathcal{N}_{2}$ denoting the nearest left and right neighbors of $m$ we have $m_{-} \leq m-1$ and $m_{+} \geq m+1$, and thus

$$
\frac{1}{(n-(m+\delta))^{2}}=\frac{1+O(\gamma)}{(n-m)^{2}}
$$

holds for all $n \in \mathcal{N}_{2} \backslash\{m\}$ and $|\delta| \leq \gamma$. Thus,

$$
\min _{|\delta| \leq \gamma} f^{\prime}(\delta)=\min _{|\delta| \leq \gamma} H_{m}^{\prime}(m+\delta)=H_{m}^{\prime}(m)(1+O(\gamma))
$$

for $|\delta| \leq \gamma$. Hence we may take $A=r_{2}(m)$ and $B=H_{m}^{\prime}\left(m+\delta_{0}\right)(1+O(\gamma))$ in Lemma 6 ; on squaring the estimate $\delta_{0} \leq \sqrt{A / B}$ we find that

$$
\delta_{0}^{2} H_{m}^{\prime}\left(m+\delta_{0}\right) \leq(1+O(\gamma)) r_{2}(m)
$$

(for $\gamma$ small). In particular, $\lambda=m+\delta_{0}$ is a new eigenvalue, and

$$
H_{m}^{\prime}(\lambda) \leq \frac{r_{2}(m)}{(m-\lambda)^{2}} \cdot(1+O(\gamma))
$$

The proof of Proposition 11 is thus concluded.

Remark 12. The above argument in fact gives the following: if $r_{2}(m+h) \geq R \cdot r_{2}(m)>0$ for some $0<h<H$, then (again for $|\delta|<1 / 10$ ),

$$
f^{\prime}(\delta) \gg \frac{R \cdot r_{2}(m)}{H^{2}}
$$

and thus there exists a nearby new eigenvalue $\lambda=m+\delta_{0}$ with $\left|\delta_{0}\right| \ll H / \sqrt{R}$, and

$$
H_{m}^{\prime}(\lambda) \leq \frac{r_{2}(m)}{(m-\lambda)^{2}}\left(1+O\left(\frac{H}{\sqrt{R}}\right)\right)
$$


For $\gamma \in(0,1 / 10)$ let $\mathcal{M}_{\gamma}$ be the set given by Lemma 7 ; given $m \in \mathcal{M}_{\gamma}$ let $\lambda_{m}$ denote the nearby new eigenvalue with the above properties (in particular, $\left|\lambda_{m}-m\right| \leq \gamma$, and (35) holds for $\lambda=\lambda_{m}$ ), and define $\Lambda_{\gamma}:=\left\{\lambda_{m}: m \in \mathcal{M}_{\gamma}\right\}$. Taking $\gamma_{k}=1 / k$ for integer $k>10$, we find that $\Lambda_{\gamma_{k+1}} \subset \Lambda_{\gamma_{k}}$, hence there exists an infinite sequence

$$
\Lambda_{0}=\left\{\lambda_{m_{1}}<\lambda_{m_{2}}<\cdots\right\}
$$

such that $\lambda_{m_{i}} \in \Lambda_{\gamma_{j}}$ for $i \geq j$.

A standard compactness argument then gives the following: there exists an infinite subsequence $\Lambda^{\prime} \subset \Lambda_{0}$ such that

$$
\Omega(m):=\left\{v /|v|: v \in \mathbb{Z}^{2},|v|^{2}=m\right\}
$$

converges to a limit multiset $\Omega(\infty)$ of bounded cardinality as $\lambda_{m} \in \Lambda^{\prime}$ tends infinity (in particular, we may assume that $|\Omega(m)|=r_{2}(m)$, is constant). Moreover, since $\lambda_{m_{i}} \in \Lambda_{\gamma_{j}}$ for $i \geq j$ if $\lambda_{m_{i}} \in \Lambda_{0}$, and we have passed to a subsequence of $\Lambda_{0}$, we find that for all $k \in \mathbb{Z}^{+}$, there exists $t_{k} \in \mathbb{Z}^{+}$such that

$$
\Lambda^{\prime} \cap\left(t_{k}, \infty\right) \subset \Lambda_{\gamma_{k}} \text {. }
$$

We may also assume that $\lim _{\lambda \rightarrow \infty, \lambda \in \Lambda^{\prime}}\left\langle\mathrm{Op}(f) g_{\lambda}, g_{\lambda}\right\rangle=v_{\infty}(f)$ for some semiclassical measure $v_{\infty}$ by passing to a further subsequence.

Remark 13. Note that a limit of sets, say of fixed cardinality, might be a multiset. E.g., in our construction, the Wigner measures have singular (momentum) support in the two directions $(n, \pm 1) / \sqrt{n^{2}+1}$; letting $n \rightarrow \infty$ we obtain a measure with support on $(1,0)$ and "twice the mass". In particular, the support set of $\Omega(\infty)$ might have smaller cardinality than $\underline{\lim }_{n}|\Omega(n)|$.

5.1. Singular momentum marginals. It is now straightforward to exhibit singular momentum marginals_-for any fixed positive $f \in C^{\infty}\left(\mathbb{S}^{1}\right)$ we show that

$$
\lim _{\lambda \rightarrow \infty, \lambda \in \Lambda^{\prime}}\left\langle\mathrm{Op}(f) g_{\lambda}, g_{\lambda}\right\rangle \geq \frac{1 / 2}{|\Omega(\infty)|} \sum_{\xi \in \Omega(\infty)} f(\xi)=\frac{1}{2} \cdot \mu_{\Omega(\infty)}(f) .
$$

Let

$$
W_{f}(n):=\sum_{|v|^{2}=n} f\left(\frac{v}{|v|}\right) .
$$

We start with an upper bound on the $L^{2}$ norm of $G_{\lambda}$ valid for $\lambda \in \Lambda^{\prime}$ and $\lambda \rightarrow \infty$. By definition of $G_{\lambda}$, its $L^{2}$ norm is [recall that $\lambda=\lambda_{m}=m+\delta$, and that $\lambda \in \Lambda_{\gamma_{k}}$ for any $k$ provided that $\lambda \in \Lambda^{\prime}$ is sufficiently large; cf. (36)]

$$
\begin{aligned}
\left\|G_{\lambda}\right\|^{2} & =\sum_{n \in \mathcal{N}_{2}} \frac{r_{2}(n)}{|n-\lambda|^{2}}=\frac{r_{2}(m)}{|m-\lambda|^{2}}+\sum_{\substack{n \in \mathcal{N}_{2} \\
n \neq m}} \frac{r_{2}(n)}{(n-\lambda)^{2}} \\
& =\frac{r_{2}(m)}{|m-\lambda|^{2}}+H_{m}^{\prime}(m+\delta)+\sum_{n \notin I(\lambda)} \frac{r_{2}(n)}{|n-\lambda|^{2}} \\
& \leq\left(2+O\left(\gamma_{k}\right)\right) \frac{r_{2}(m)}{|m-\lambda|^{2}}+O\left(\frac{\lambda^{\epsilon}}{\lambda^{\eta}}\right)=(2+o(1)) \frac{r_{2}(m)}{|m-\lambda|^{2}}+o(1)
\end{aligned}
$$


as $\lambda \rightarrow \infty$, where the last inequality follows from Proposition 11, and that $r_{2}(n) \ll_{\varepsilon} n^{\varepsilon}$.

Recalling that $f$ is positive (and that $|m-\lambda|=O(1)$ ), this implies that, as $\lambda \rightarrow \infty$,

$$
\begin{aligned}
\left\langle\mathrm{Op}(f) g_{\lambda}, g_{\lambda}\right\rangle & =\frac{\sum_{n \in \mathcal{N}_{2}} \frac{W_{f}(n)}{(n-\lambda)^{2}}}{\sum_{n \in \mathcal{N}_{2}} \frac{r_{2}(n)}{(n-\lambda)^{2}}} \geq \frac{\frac{W_{f}(m)}{(m-\lambda)^{2}}}{(2+o(1)) \frac{r_{2}(m)}{(m-\lambda)^{2}}+o(1)} \\
& =\frac{1}{2+o(1)} \cdot \frac{W_{f}(m)}{r_{2}(m)+o(1)} \\
& =\frac{1+o(1)}{2 \cdot|\Omega(\infty)|} \sum_{\xi \in \Omega(\infty)} f(\xi)=\frac{1+o(1)}{2} \cdot \mu_{\Omega(\infty)}(f) .
\end{aligned}
$$

Remark 14. We note that the above construction places mass at least $1 / 2$ on the (momentum) singular part.

Remark 15. A slightly more general statement follows by the same arguments used above. Namely, given $M, R, H>0$, suppose that $\mathcal{M} \subset \mathcal{N}_{2}$ is a subset such that for all $m \in \mathcal{M}, r_{2}(m)<M$, and $r_{2}(m+h)>R \cdot r_{2}(m)$ for some integer $h \in(0, H)$. If we let $\Lambda(\mathcal{M})$ be the corresponding set of new eigenvalues given by Remark 12, we then have

$$
\begin{aligned}
\left\langle\mathrm{Op}(f) g_{\lambda}, g_{\lambda}\right\rangle & \geq \frac{1 / 2}{|\Omega(m)|} \sum_{\xi \in \Omega(m)} f(\xi)\left(1+O\left(\frac{H}{\sqrt{R}}\right)\right) \\
& =\frac{1}{2} \cdot \mu_{\Omega(m)}(f)\left(1+O\left(\frac{H}{\sqrt{R}}\right)\right), \quad \text { for } \lambda \in \Lambda(\mathcal{M}) .
\end{aligned}
$$

5.2. Non-uniform quantum limits. To simplify the notation, we use the following convention throughout this section: let $w:=(0,2) \in \mathbb{Z}^{2}$, and for $\lambda$ a fixed "new" eigenvalue, and $v \in \mathbb{Z}^{2}$ define

$$
c(v):=c_{\lambda}(v)=\frac{1}{|v|^{2}-\lambda}, \quad C(v, w):=c(v) c(v+w) .
$$

By the definition of $G_{\lambda}$ and $\operatorname{Op}(a)$ we see that using the new notation (cf. (9), (12))

$$
\mathrm{Op}\left(e_{w}\right) G_{\lambda}\left(x, x_{0}\right)=\sum_{v \in \mathbb{Z}^{2}} c(v) e^{i v \cdot x_{0}} e^{i(v+w) \cdot x},
$$

and therefore

$$
\left\langle\mathrm{Op}\left(e_{w}\right) g_{\lambda}, g_{\lambda}\right\rangle=\frac{e^{-i w \cdot x_{0}} \cdot \sum_{v \in \mathbb{Z}^{2}} c(v) c(v+w)}{\sum_{m \in \mathcal{N}_{2}} \frac{r_{2}(m)}{(m-\lambda)^{2}}}=\frac{e^{-i w \cdot x_{0}} \cdot \sum_{v \in \mathbb{Z}^{2}} C(v, w)}{\sum_{m \in \mathcal{N}_{2}} \frac{r_{2}(m)}{(m-\lambda)^{2}}}
$$

As we aim to show that (41) is bounded from below in absolute value, we assume that $x_{0}=0$ for simplicity. We will show that the sum in the numerator is essentially bounded from below by two terms in the sum, namely $v$ such $|v|=|v+w|$.

In what follows, $\lambda=\lambda_{m}=m+\delta \in \Lambda^{\prime}$ (where $|\delta| \leq \gamma_{k}=1 / k=o(1)$ as $\lambda \rightarrow \infty$ ), and $m=n^{2}+1$ for $n \in \mathbb{Z}^{+}$. Given such an $n$ we define a vector $u \in \mathbb{Z}^{2}$, with $|u|^{2}=m$, by

$$
u:=(n,-1)
$$



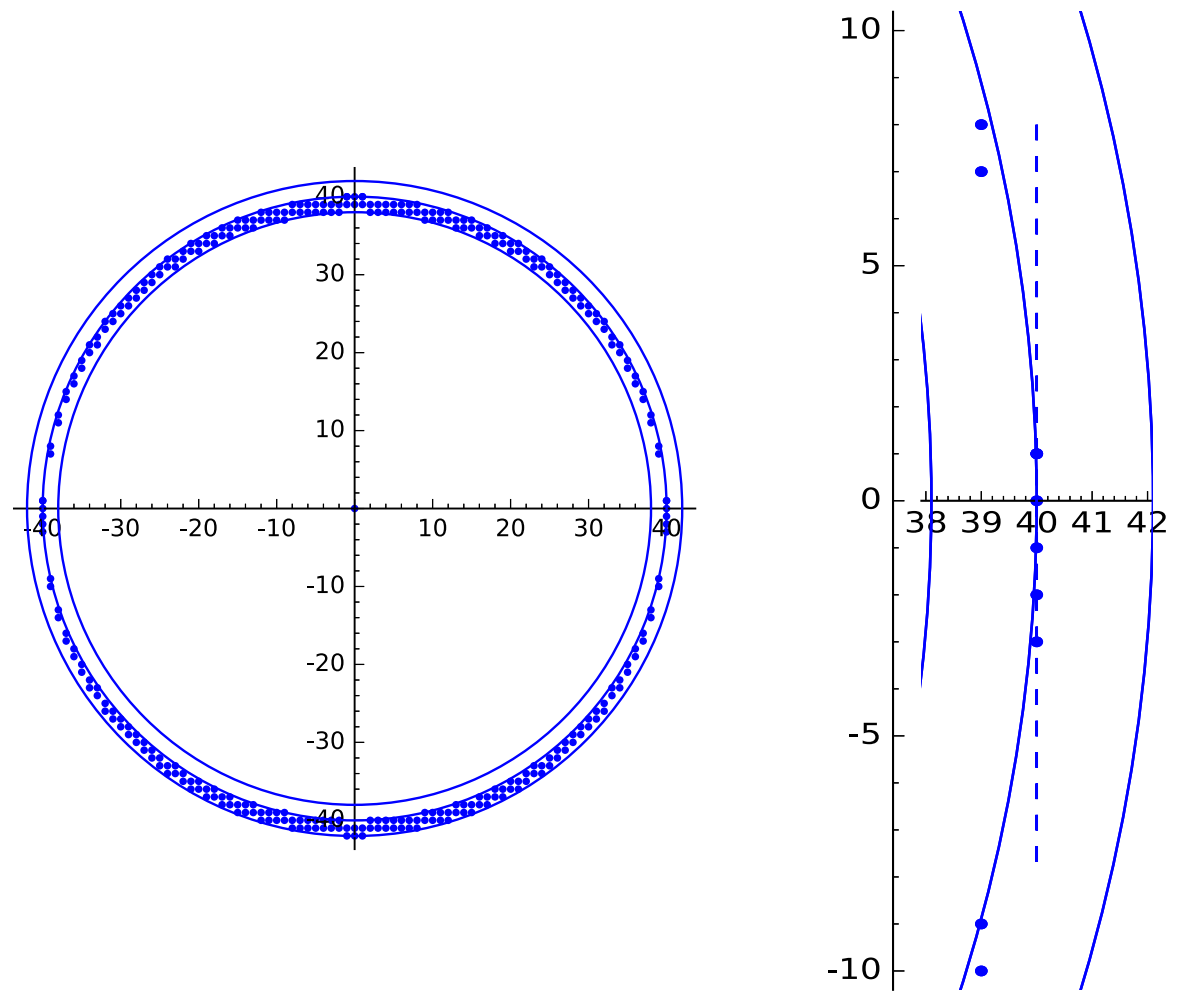

Fig. 1. An illustration of the setting of Lemma 16. For $m=40^{2}+1$ and (say) $\delta=0.1$, only the lattice points with $C(v, w)<0$ and the points $( \pm 40, \pm 1)$ are plotted. On the right plot we zoomed around the point $(40,0)$. Notice that the only points near $(40, \pm 1)$ lie on the line $x=40$. We remark that the condition that $|\langle v, w\rangle| \leq \sqrt{R}$ in Lemma 16 here corresponds to $|y| \leq \sqrt{40} / 2 \sim 3.166$

For notational convenience, set $R=\sqrt{\lambda}$, and let

$$
C_{m}:=\left\{v \in \mathbb{R}^{2}:|v|^{2}=m\right\}
$$

denote the circle of radius $\sqrt{m}$ centered at the origin. Define

$$
A_{R}=A_{R, w}:=\left\{v \in \mathbb{R}^{2}:|v| \in[R-|w|, R+|w|]\right\}
$$

as the annulus of width $2|w|$ containing $C_{m}$, and let

$$
A_{R}^{*}=A_{R, w}^{*}:=\left\{v \in \mathbb{R}^{2}:|v| \in[R-|w|, R+|w|],|v|^{2} \neq m,|v+w|^{2} \neq m\right\} .
$$

The following Lemma will allow us to bound the contribution of the negative terms in the sum in the numerator of the right hand side of (41). We refer the reader to Fig. 1 for an illustration of the following Lemma.

Lemma 16. If $C(v, w)<0$ for $v \in \mathbb{Z}^{2}$, then $|v| \in[R-|w|, R+|w|]$. Furthermore, if we in addition have $|\langle v, w\rangle| \leq \sqrt{R}$, then $v=( \pm n, y)$ with $-3 \leq y \leq 1$ provided that $R$ is sufficiently large. 
Proof. Since $C(v, w)<0$ if and only if the line segment joining $v$ and $v+w$ intersects $C_{R}$, the first assertion follows from the triangle inequality.

We now write $v=(x, y)$ for $x, y \in \mathbb{Z}$.

First case: If $|x| \geq n+1$, then

$$
|v|^{2}-\lambda \geq x^{2}-\lambda \geq(n+1)^{2}-\lambda=n^{2}+2 n+1-\lambda \geq m+1-\lambda \geq 1-\delta
$$

and similarly $|v+w|^{2}-\lambda \geq 1-\delta$. Recalling that $|\delta|<\gamma \leq 1 / 10$ we find that $C(v, w)>0$.

Second case: Assume that $|x| \leq n-1$. We note that $C(v, w)<0$ implies that either $|v|^{2}>\lambda$, or that $|v+w|^{2}>\lambda$.

Now, if $|v|^{2}>\lambda$, then

$$
|v|^{2}=x^{2}+y^{2}>\lambda=m+\delta=n^{2}+1+\delta
$$

so,

$$
y^{2}>n^{2}+1+\delta-(n-1)^{2} \geq n .
$$

Consequently, $|y| \geq \sqrt{n}>\sqrt{R} / 2$, hence $|\langle v, w\rangle|=2|y|>\sqrt{R}$ and the claim is vacuous.

On the other hand, if $|v+w|^{2}>\lambda$ then, as $x^{2} \leq(n-1)^{2}$,

$$
|v+w|^{2}=x^{2}+(y+2)^{2}>\lambda=m+\delta=n^{2}+1+\delta
$$

so $|y| \geq \sqrt{n}$, and as before $|\langle v, w\rangle|>\sqrt{R}$; again the claim is vacuous.

Third case: For $|x|=n$, since $|\delta|=|\lambda-m|<1 / 10$ we find that

$$
|v|^{2}-\lambda=n^{2}+y^{2}-\lambda=m-\lambda+y^{2}-1=-\delta+y^{2}-1,
$$

and

$$
|v+w|^{2}-\lambda=n^{2}+(y+2)^{2}-\lambda=-\delta+(y+2)^{2}-1
$$

so both $c(v), c(v+w)$ are positive for $v=( \pm n, y)$ if $y \leq-4$ or $y \geq 2$.

In light of Lemma 16, we consider the following three sets of points $v \in \mathbb{Z}^{2}$ :

$$
\begin{aligned}
& V_{1}:=\left\{v \in \mathbb{Z}^{2}: v=( \pm n, y),-3 \leq y \leq 1\right\} \\
& V_{2}:=\left\{v \in \mathbb{Z}^{2}: C(v, w)<0, \frac{|v|^{2},|v+w|^{2} \neq m,}{\sqrt{R} \leq|\langle v, w\rangle| \leq 3 R}\right\} \\
& V_{3}:=\left\{v \in \mathbb{Z}^{2}: C(v, w)<0, \begin{array}{c}
|v|^{2}=m \text { or }|v+w|^{2}=m, \\
\sqrt{R} \leq|\langle v, w\rangle| \leq 3 R
\end{array}\right\} .
\end{aligned}
$$

Notice that these three sets, for $R$ sufficiently large, cover all $v \in \mathbb{Z}^{2}$ such that $C(v, w)<0$, because if $C(v, w)<0$, then $|\langle v, w\rangle| \leq(R+|w|)|w|<3 R$ for $R>4$. Before we proceed to the rest of the proof, we explain first how the contribution from each set is treated. We also refer the reader to Fig. 3 for an illustration of the case of $v \in V_{2}$, and to Fig. 2 for the case of $v \in V_{1}$. For $v \in V_{1}$, we show that the main contribution of $C(v, w)$ comes from $v=( \pm n,-1)$ where $c(v)=c(v+w)=1 / \delta$, and for the other finite members either $c(v)$ or $c(v+w)$ is bounded, and hence the contribution from these is bounded by $\ll 1 / \delta$. For $v \in V_{3}$, although either $c(v)$ or $c(v+w)$ equals $1 / \delta$, the second term is bounded by $\ll 1 / R$, and so the contribution from all summands 


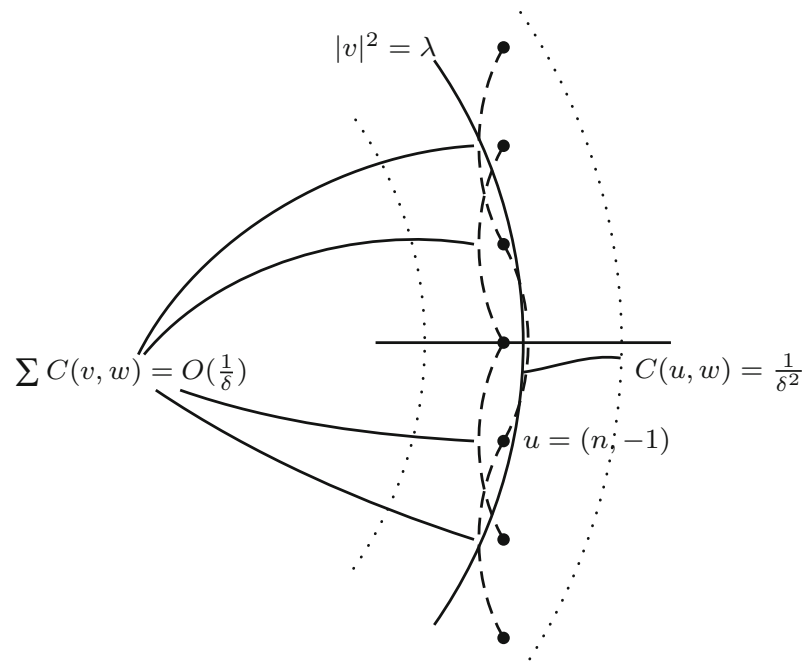

Fig. 2. The main contribution in Corollary 17 is seen here. The dashed lines connect points differing by $w=(0,2)$. For $v=u$ we have that $C(u, w)=c(u) c(u+w)=\frac{1}{\delta^{2}}$, and all other points $v$ with $C(v, w)<0$ contribute $O\left(\frac{1}{\delta}\right)$

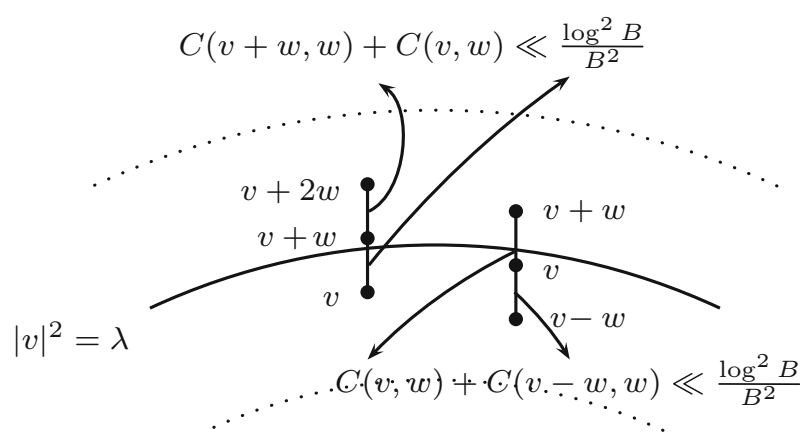

Fig. 3. An illustration of the "pairing off" in Lemma 18. For $v \in V_{2}$, at least one of the terms $C(v, w)+C(v+$ $w, w)$ or $C(v, w)+C(v-w, w)$ is $\ll \frac{\log ^{2} B}{B^{2}}$

is $\ll 1 /(R \delta)$. The most subtle treatment is for $v \in V_{2}$ : here neither $c(v)$ nor $c(v+w)$ are as large as $1 / \delta$, but as there are many summands we need some further cancellation. To obtain this, when needed, we "pair off" negative terms $C(v, w)$ with "nearby" positive terms (see Fig. 3).

For ease of notation, we make the following definitions: For a finite set $X \subset \mathbb{R}$, define $\operatorname{argmin}_{X}(|x|)$ as the leftmost $x \in X$ which minimizes $|x|$, and for $v \in \mathbb{Z}^{2}$ and $w=(0,2)$ as before, let

$$
\begin{aligned}
\operatorname{Nbr}_{w}(v) & :=\{C(v, w), C(v-w, w)+C(v, w), C(v, w)+C(v+w, w)\} \\
S_{w}(v) & :=\operatorname{argmin}_{\mathrm{Nbr}_{w}(v)}(|x|) .
\end{aligned}
$$


Corollary 17. For $\lambda=m+\delta$ and $|\delta|$ small enough, as $\lambda \rightarrow \infty$, we have

$$
\sum_{v \in \mathbb{Z}^{2}} C(v, w) \geq \frac{2}{\delta^{2}}+O\left(\frac{1}{\delta}\right)+\sum_{v \in V_{2}} S_{w}(v)
$$

Proof. We first notice that if $|t| \leq \frac{|\langle v, w\rangle|}{|w|^{2}}$, then only one sign change can occur for $|v+t w|^{2}-\lambda$. In particular, for $\lambda$ large enough (and hence also $R=\sqrt{\lambda}$ ), if $v \in V_{2} \cup V_{3}$ and $C(v, w)<0$ then both $C(v-w, w), C(v+w, w)>0$. Also, as mentioned above, by Lemma 16, if $C(v, w)<0$ then $v$ is in either $V_{1}, V_{2}$ or $V_{3}$ for $R$ large. Therefore, after removing only positive terms, we find that

$$
\begin{aligned}
\sum_{v \in \mathbb{Z}^{2}} C(v, w) & \geq \sum_{v \in V_{1}} C(v, w)+\sum_{v \in V_{3}} C(v, w)+\sum_{v \in V_{2}} S_{w}(v) \\
& =2 \sum_{y=-3}^{1} C((n, y), w)+\sum_{v \in V_{3}} C(v, w)+\sum_{v \in V_{2}} S_{w}(v)
\end{aligned}
$$

Now, by definition of $C(v, w)$, for the first sum we have that

$$
\begin{aligned}
& \sum_{y=-3}^{1} C(( \pm n, y), w) \\
& \quad=\frac{1}{(-\delta)^{2}}+2\left(\frac{1}{(3-\delta)(-1-\delta)}-\frac{1}{(8-\delta) \delta}\right)=\frac{1}{\delta^{2}}+O\left(\frac{1}{\delta}\right) .
\end{aligned}
$$

(note that when $\delta \rightarrow 0$, the dominant term $1 / \delta^{2}$ comes from the term $y=-1$ ).

For $v \in V_{3}$ and $|v|^{2}=m$, we have $|\langle v, w\rangle| \geq \sqrt{R}$, and so (recall that $m-\lambda=-\delta$ )

$$
|| v+\left.w\right|^{2}-\lambda|=||v|^{2}+2\langle v, w\rangle+|w|^{2}-\lambda|=| 2\langle v, w\rangle+|w|^{2}-\delta \mid \gg \sqrt{R} .
$$

and $C(v, w) \ll \frac{1}{\delta \sqrt{R}}$. Using a similar argument we get that $C(v, w) \ll \frac{1}{\delta \sqrt{R}}$ if $|v+w|^{2}=$ $m$. Therefore, since $r_{2}(m)$ is bounded,

$$
\sum_{v \in V_{3}} C(v, w) \ll \sum_{v \in V_{3}} \frac{1}{\delta \sqrt{R}} \ll \frac{1}{\delta \sqrt{R}}
$$

and (42) follows.

The contribution from $v \in V_{2}$ is more subtle, and those $v$ for which $C(v, w)$ is not very small are treated by pairing off negative summands with "nearby" positive ones, thus getting some extra savings.

Lemma 18. If $v \in V_{2}$, then $S_{v}(w) \ll \frac{\log ^{2}|\langle v, w\rangle|}{|\langle v, w\rangle|^{2}}$ as $R \rightarrow \infty$.

Proof. For notational convenience, we put $B:=B_{v}=|\langle v, w\rangle|$. After recalling that $v \in V_{2}$ implies that $|\langle v, w\rangle| \geq R^{1 / 2}$, we split the proof into two cases.

First case: Here we assume that $\left.|| v\right|^{2}-\lambda|\leq||v+w|^{2}-\lambda \mid$. If $\left.|| v\right|^{2}-\lambda \mid \geq B / \log B$, then

$$
|C(v, w)|=|c(v) c(v+w)| \leq \frac{\log ^{2} B}{B^{2}} .
$$


and there is no need for "pairing off" the negative term with a positive one. We may therefore assume that $\left.|| v\right|^{2}-\lambda \mid \leq B / \log B$. Now,

$$
|v+w|^{2}-\lambda=|v|^{2}+2\langle v, w\rangle+|w|^{2}-\lambda=|v|^{2}-\lambda \pm 2 B+|w|^{2}
$$

and similarly $|v-w|^{2}-\lambda=|v|^{2}-\lambda-( \pm 2 B)+|w|^{2}$, hence

$$
\begin{aligned}
C(v, w)+C(v-w, w) & =c(v) \cdot c(v+w)+c(v-w) \cdot c(v) \\
& =\frac{1}{|v|^{2}-\lambda}\left(\frac{2\left(|v|^{2}+|w|^{2}-\lambda\right)}{\left(|v|^{2}+2 B+|w|^{2}-\lambda\right)\left(|v|^{2}-2 B+|w|^{2}-\lambda\right)}\right) .
\end{aligned}
$$

(note that the two $\pm 2 B$ terms above occur with opposite signs). Recalling the assumption $|v|^{2} \neq m$, together with $|w|^{2}=4$, we find that $\left.|| v\right|^{2}-\lambda \mid \geq 1 / 2$ (note that $|\lambda-m| \leq$ $\delta \leq 1 / 10$ by our assumption on $\delta$ ), and this together with (45) shows that

$$
C(v, w)+C(v-w, w) \ll \frac{1}{\left(|v|^{2}+2 B+|w|^{2}-\lambda\right)\left(|v|^{2}-2 B+|w|^{2}-\lambda\right)} .
$$

Since we assume that $\left.|| v\right|^{2}-\lambda \mid \leq B / \log B$, we find that

$$
\left.|| v\right|^{2} \pm 2 B+|w|^{2}-\lambda \mid \gg B
$$

and thus $C(v, w)+C(v-w, w) \ll 1 / B^{2}$.

Second case Here we assume that $\left.|| v\right|^{2}-\lambda|>||v+w|^{2}-\lambda \mid$. This case follows by a similar argument, except for showing that

$$
|c(v+w)(c(v)+c(v+2 w))| \ll \frac{\log ^{2} B}{B^{2}} .
$$

Corollary 19. As $R \rightarrow \infty$, we have

$$
\sum_{v \in V_{2}} S_{w}(v)=\sum_{\substack{v \in A_{R}^{*} \\ C(v, w)<0 \\|\langle v, w\rangle| \in\left[R^{1 / 2}, 3 R\right]}} S_{w}(v)=o(1) .
$$

Proof. Write

$$
=\sum_{\substack{v \in A_{R}^{*} \\ C(v, w)<0 \\|\langle v, w\rangle| \in\left[R^{1 / 2}, 3 R\right]}} S_{w}(v)
$$

Since the number of lattice points in $A_{R}$ satisfying $\langle v, w\rangle \in I$ is $O(|I||w|)$ for any interval $I \subset[-R / \log R, R / \log R]$, we get by Lemma 18 that

$$
\sum_{\substack{k \in \mathbb{N} \\ R^{1 / 2} \leq 2^{k}<R / \log R}} \sum_{\substack{v \in A_{R}^{*} \\ C(v, w)<0 \\|\langle v, w\rangle| \in\left[2^{k}, 2^{k+1}\right)}} S_{w}(v) \ll \sum_{2^{k} \geq R^{1 / 2}} 2^{k}\left(\frac{k}{2^{k}}\right)^{2} \ll \frac{1}{R^{1 / 2-\varepsilon}}=o(1)
$$


and

$$
\sum_{\substack{v \in A_{R}^{*} \\ C(v, w)<0 \\ \mid \in[R / \log R, 3 R]}} S_{w}(v) \ll R \frac{\log ^{2} R}{(R / \log R)^{2}}=\frac{\log ^{4} R}{R}=o(1)
$$

5.2.1. Conclusion. We can now prove localization in position by proving that for $f(x)=$ $e^{i\langle x, w\rangle} \in C^{\infty}\left(\mathbb{T}^{2}\right)$,

$$
\lim _{\lambda \in \Lambda^{\prime}}\left\langle\mathrm{Op}(f) g_{\lambda}, g_{\lambda}\right\rangle=c>0
$$

By (41) (recall that we assumed that $x_{0}=0$ ),

$$
\left\langle O p\left(e_{w}\right) g_{\lambda}, g_{\lambda}\right\rangle=\frac{\sum_{v \in \mathbb{Z}^{2}} C(v, w)}{\sum_{m \in \mathcal{N}_{2}} \frac{r_{2}(m)}{(m-\lambda)^{2}}}
$$

Recalling the notational convention that $\lambda=\lambda_{m}=m+\delta$, where $|\delta| \leq \gamma$, corollaries 17 and 19 gives that

$$
\sum_{v \in \mathbb{Z}^{2}} C(v, w) \geq \frac{2}{\delta^{2}}+O\left(\frac{1}{\delta}\right)+o(1) \geq \frac{2}{\gamma^{2}}+O\left(\frac{1}{\gamma}\right)+o(1)
$$

On the other hand, by Proposition 11,

$$
\sum_{n \in \mathcal{N}_{2}} \frac{r_{2}(n)}{(n-\lambda)^{2}}=\frac{r_{2}(m)}{(m-\lambda)^{2}}+H_{m}^{\prime}(\lambda) \leq(2+O(\gamma)) \frac{r_{2}(m)}{(m-\lambda)^{2}}
$$

and, on recalling that $r_{2}(m)$ is bounded, we find that for $\lambda \in \Lambda^{\prime}$ tending to infinity (and hence $\gamma=o(1)$ since $\lambda \in \Lambda^{\prime}$ implies that $\lambda \in \Lambda_{\gamma_{k}}$ for any fixed $k$ if $\lambda$ is sufficiently large), we have

$$
\left\langle\mathrm{Op}\left(e_{w}\right) g_{\lambda}, g_{\lambda}\right\rangle \geq \frac{\frac{2}{\gamma^{2}}+O\left(\frac{1}{\gamma}\right)}{(2+O(\gamma)) \frac{r_{2}(m)}{\gamma^{2}}}=\frac{1+o(1)}{r_{2}(m)}
$$

which is uniformly bounded from below.

Remark 20. Since the set $\left\{v \in \mathbb{Z}^{2}:|v|^{2}=m\right\}$ is invariant under $v \rightarrow-v$, and $v=(a, b) \rightarrow v^{\prime}=(b, a)$, the bound $\left\langle\mathrm{Op}\left(e_{w}\right) g_{\lambda}, g_{\lambda}\right\rangle \geq \frac{1+o(1)}{r_{2}(m)}$ holds for $w \in\{(0, \pm 2),( \pm 2,0)\}$.

We also note that the method of proof, with minor modifications, works for any $w \in \mathbb{Z}^{2}$ such that $|w|^{2}$ is even. 


\section{Proof of Theorem 3}

Recall first the setting proved in Proposition 9: There exist an integer $H \geq 1$ with the property that for all sufficiently large $R$ there exist an integer $h \in\left(0, H^{2}\right)$ such that

$$
\left|\left\{n \in \mathcal{N}_{2}: n \leq x, 0<r_{2}(n) \ll R^{H}, r_{2}(n+h) \geq R \cdot r_{2}(n)\right\}\right| \gg_{R} x /(\log x)^{H}
$$

as $x \rightarrow \infty$.

As noted in Remark 12, if $r_{2}(m+h) \geq R \cdot r_{2}(m)>0$ for some integer $h$ such that $0<h<H$, then there exists a new eigenvalue $\lambda=\lambda_{m}=m+\delta_{0}$ with

$$
\delta_{0} \ll 2 H / \sqrt{R}, \quad H_{m}^{\prime}(\lambda) \ll \frac{r_{2}(m)}{(m-\lambda)^{2}} .
$$

Let $\Lambda^{\prime \prime}=\left\{\lambda_{m}\right\}$ denote the sequence of new eigenvalues with the above property. Proposition 9 then gives, upon choosing $R$ sufficiently large, that the number of such $m \leq x$ is $\gg x /(\log x)^{H}$, and the same holds for the counting function for $\Lambda^{\prime \prime}$.

Now, if $\Lambda^{\prime \prime \prime} \subset \Lambda^{\prime \prime}$ denotes any subset along which the semi-classical limit exists, the argument in Sect. 5.1 shows, as mentioned in Remark 15 that the limit as $\lambda \in \Lambda^{\prime \prime \prime}$ tends to infinity gives rise to singular momentum marginals provided $r_{2}(m)$ is also bounded; by the above construction $r_{2}(m) \ll R^{H}$.

\section{A. Uniform Quantum Limits in Theorem 3}

As before, given a nonzero $w \in \mathbb{Z}^{2}$ it is enough to show the existence of a subset $\Lambda_{w}^{\prime \prime} \subset \Lambda^{\prime \prime}$, of full relative density, such that

$$
\lim _{\lambda \rightarrow \infty, \lambda \in \Lambda_{w}^{\prime \prime}}\left\langle\mathrm{Op}\left(e_{w, k}\right) g_{\lambda}, g_{\lambda}\right\rangle=0
$$

where $e_{w, k}(x, \xi)=e^{i\langle w, x\rangle} \cdot e^{2 \pi i k \xi}$. More precisely, letting

$$
\left.\Lambda_{\text {flat }}^{\prime \prime}:=\Lambda^{\prime \prime} \backslash\left(\cup_{w \in \mathbb{Z}^{2} \backslash\{0\}}\left(\Lambda^{\prime \prime} \backslash \Lambda_{w}^{\prime \prime}\right) \cap\left[t_{w}, \infty\right]\right)\right),
$$

we obtain a subset $\Lambda_{\text {flat }}^{\prime \prime} \subset \Lambda^{\prime \prime}$ which has full relative density provided that we let $t_{w} \in \mathbb{Z}^{+}$ grow sufficiently quickly as $|w| \rightarrow \infty$.

Following the arguments in [35] we define $\Lambda_{w}^{\prime \prime} \subset \Lambda^{\prime \prime}$ as follows. Fix $\theta \in(0,1 / 3)$ and $\delta \in(\theta / 2,1 / 2-\theta)$. Given $0 \neq w \in \mathbb{Z}^{2}$, let

$$
S_{w}:=\left\{v \in \mathbb{Z}^{2}:|\langle v, w\rangle| \leq|v|^{2 \delta}\right\} .
$$

Further, define the annulus

$$
A(\lambda, L):=\left\{v \in \mathbb{Z}^{2}:\left.|| v\right|^{2}-\lambda \mid<L\right\},
$$

and put

$$
\Lambda_{w}^{\prime \prime}:=\left\{\lambda \in \Lambda^{\prime \prime}: A\left(\lambda, \lambda^{\delta}\right) \cap S_{w}=\emptyset\right\}
$$

To conclude the proof, we next show that $\Lambda_{w}^{\prime \prime} \subset \Lambda^{\prime \prime}$ indeed has the desired properties (recall that $\left|\left\{\lambda \in \Lambda^{\prime \prime}: \lambda<X\right\}\right| \gg X /(\log X)^{A}$ for some $\left.A>1\right)$. 
Proposition 21. There exists $\delta^{\prime}>0$ such that for all $w \neq 0$

$$
\left|\left\{\lambda \in \Lambda^{\prime \prime} \backslash \Lambda_{w}^{\prime \prime}: \lambda \leq X\right\}\right| \ll X^{1-\delta^{\prime}} /|w|
$$

as $X \rightarrow \infty$. Moreover, as $\lambda \in \Lambda_{w}^{\prime \prime}$ tends to infinity,

$$
\left|\left\langle\mathrm{Op}\left(e_{w, k}\right) g_{\lambda}, g_{\lambda}\right\rangle\right| \rightarrow 0 .
$$

Proof. Take $L=\lambda^{\delta}$ and define a truncated Green's function

$$
G_{\lambda, L}(x):=\sum_{v \in A(\lambda, L)} \frac{e^{i v \cdot x}}{|v|^{2}-\lambda}
$$

and its $L^{2}$ normalization $g_{\lambda, L}:=G_{\lambda, L} /\left\|G_{\lambda, L}\right\|_{2}$. By Lemmas 5.1 and 5.2 in [35], we then have $\left\|g_{\lambda}-g_{\lambda, L}\right\|_{2} \rightarrow 0$ and

$$
\left|\left\langle\mathrm{Op}\left(e_{w, k}\right) g_{\lambda}, g_{\lambda}\right\rangle-\left\langle\mathrm{Op}\left(e_{w, k}\right) g_{\lambda, L}, g_{\lambda, L}\right\rangle\right| \rightarrow 0
$$

as $\lambda \in \Lambda_{w}^{\prime \prime}$ tends to infinity. (Note that $\lambda \in \Lambda_{w}^{\prime \prime} \subset \Lambda^{\prime \prime}$ implies that $\left\|G_{\lambda}\right\|_{2}^{2} \geq r_{2}(m) /(m-$ $\left.\lambda)^{2} \gg 1\right)$. It is thus enough to show that

$$
\left|\left\langle\mathrm{Op}\left(e_{w, k}\right) g_{\lambda, L}, g_{\lambda, L}\right\rangle\right| \rightarrow 0
$$

for $\lambda \in \Lambda_{w}$ tending to infinity. Since $w \neq 0,[27$, Section 2.2] implies that

$$
\begin{aligned}
\left|\left\langle\mathrm{Op}\left(e_{w, k}\right) g_{\lambda, L}, g_{\lambda, L}\right\rangle\right| & \leq \sum_{v \in \mathbb{Z}^{2}}\left|\widehat{g_{\lambda, L}}(v) \cdot \widehat{g_{\lambda, L}}(v-w)\right| \\
& \ll \frac{1}{\left\|G_{\lambda, L}\right\|_{2}^{2}} \sum_{v \in A(\lambda, L)} \frac{1}{\left.|| v\right|^{2}-\lambda \mid} \cdot \frac{1}{|| v-\left.w\right|^{2}-\lambda \mid}
\end{aligned}
$$

which, by the proof of [35, Proposition 7.1], tends to zero.

Finally, (49) follows from [35, Equation 6.4].

Acknowledgements. First we would like to thank Zeév Rudnick and Henrik Ueberschär for helpful discussions about this work. Secondly, we wish to thank the anonymous referees for many useful comments and suggestions how to improve the paper; in particular strengthening Theorems 1 and 3 by going beyond momentum marginals. Funding was provided by Göran Gustafssons Stiftelse för Naturvetenskaplig och Medicinsk Forskning and Vetenskapsrådet (grant no. 621-2011-5498).

Open Access This article is distributed under the terms of the Creative Commons Attribution 4.0 International License (http://creativecommons.org/licenses/by/4.0/), which permits unrestricted use, distribution, and reproduction in any medium, provided you give appropriate credit to the original author(s) and the source, provide a link to the Creative Commons license, and indicate if changes were made.

\section{References}

1. Anantharaman, N., Nonnenmacher, S.: Entropy of semiclassical measures of the Walsh-quantized baker's map. Ann. Henri Poincaré 8(1), 37-74 (2007)

2. Anantharaman, N., Nonnenmacher, S.: Half-delocalization of eigenfunctions for the Laplacian on an Anosov manifold. Ann. Inst. Fourier (Grenoble) 57(7), 2465-2523 (2007) (Festival Yves Colin de Verdière)

3. Bäcker, A., Schubert, R., Stifter, P.: On the number of bouncing ball modes in billiards. J. Phys. A: Math. Gen. 30(19), 6783 (1997) 
4. Berkolaiko, G., Keating, J., Winn, B.: No quantum ergodicity for star graphs. Commun. Math. Phys. 250(2), 259-285 (2004)

5. Berkolaiko, G., Keating, J.P., Winn, B.: Intermediate wave function statistics. Phys. Rev. Lett. 91, 134103 (2003)

6. Bogomolny, E., Gerland, U., Schmit, C.: Singular statistics. Phys. Rev. E (3) 63(3, part 2), 036206, 16 (2001)

7. Bogomolny, E., Schmit, C.: Structure of wave functions of pseudointegrable billiards. Phys. Rev. Lett. 92, 244102 (2004)

8. Colin de Verdière, Y.: Ergodicité et fonctions propres du laplacien. Commun. Math. Phys. 102(3), 497$502(1985)$

9. Duke, W.: Hyperbolic distribution problems and half-integral weight Maass forms. Invent. Math. 92(1), 7390 (1988)

10. Duke, W., Schulze-Pillot, R.: Representation of integers by positive ternary quadratic forms and equidistribution of lattice points on ellipsoids. Invent. Math. 99(1), 49-57 (1990)

11. Fainsilber, L., Kurlberg, P., Wennberg, B.: Lattice points on circles and discrete velocity models for the Boltzmann equation. SIAM J. Math. Anal. 37(6), 1903-1922 (2006) (electronic)

12. Faure, F., Nonnenmacher, S.: On the maximal scarring for quantum cat map eigenstates. Commun. Math. Phys. 245(1), 201-214 (2004)

13. Faure, F., Nonnenmacher, S., De Bièvre, S.: Scarred eigenstates for quantum cat maps of minimal periods. Commun. Math. Phys. 239(3), 449-492 (2003)

14. Freiberg, T., Kurlberg, P., Rosenzweig, L.: Poisson distribution for gaps between sums of two squares and level spacings for toral point scatterers (in preparation)

15. Friedlander, J., Iwaniec, H.: Opera de cribro, vol. 57, American Mathematical Society Colloquium Publications. American Mathematical Society, Providence (2010)

16. Golubeva, E.P., Fomenko, O.M.: Asymptotic distribution of lattice points on the three-dimensional sphere. Zap. Nauchn. Sem. Leningrad. Otdel. Mat. Inst. Steklov. (LOMI), 160(Anal. Teor. Chisel i Teor. Funktsii. 8), pp. 54-71, 297 (1987)

17. Griffin, J.: On the phase-space distribution of Bloch eigenmodes for periodic point scatterers. J. Math. Phys. 57, 082101 (2016). doi:10.1063/1.4960469

18. Grosswald, E.: Representations of Integers as Sums of Squares. Springer, New York (1985)

19. Halberstam, H., Richert, H.-E.: Sieve Methods. London Mathematical Society Monographs, No. 4. Academic Press, A subsidiary of Harcourt Brace Jovanovich, Publishers, London, New York (1974)

20. Hassell, A.: Ergodic billiards that are not quantum unique ergodic. Ann. Math. (2) 171(1), 605-619 (2010) (with an appendix by the author and Luc Hillairet)

21. Jakobson, D.: Quantum limits on flat tori. Ann. Math. (2) 145(2), 235-266 (1997)

22. Keating, J.P., Marklof, J., Winn, B.: Localized eigenfunctions in Šeba billiards. J. Math. Phys. 51(6), 062101, 19 (2010)

23. Kelmer, D.: Arithmetic quantum unique ergodicity for symplectic linear maps of the multidimensional torus. Ann. Math. (2) 171(2), 815-879 (2010)

24. Kelmer, D.: Scarring for quantum maps with simple spectrum. Compos. Math. 147(5), 1608-1612 (2011)

25. Kurlberg, P., Rudnick, Z.: Hecke theory and equidistribution for the quantization of linear maps of the torus. Duke Math. J. 103(1), 47-77 (2000)

26. Kurlberg, P., Ueberschär, H.: Superscars in the seba billiard. J. Eur. Math. Soc. (JEMS) (to appear)

27. Kurlberg, P., Ueberschär, H.: Quantum ergodicity for point scatterers on arithmetic tori. Geom. Funct. Anal. 24(5), 1565-1590 (2014)

28. Lindenstrauss, E.: Invariant measures and arithmetic quantum unique ergodicity. Ann. Math. (2) 163(1), 165-219 (2006)

29. Löck, S., Bäcker, A., Ketzmerick, R.: Coupling of bouncing-ball modes to the chaotic sea and their counting function. Phys. Rev. E 85, 016210 (2012)

30. Marklof, J., Rudnick, Z.: Quantum unique ergodicity for parabolic maps. Geom. Funct. Anal. 10(6), 15541578 (2000)

31. Maynard, J.: Dense clusters of primes in subsets. Compos. Math. 152(7), 1-38 (2016)

32. Rivière, G.: Entropy of semiclassical measures in dimension 2. Duke Math J. 155(2), 271-336 (2010)

33. Rosenzweig, L.: Quantum unique ergodicity for maps on the torus. Ann. Henri Poincaré 7(3), 447469 (2006)

34. Rudnick, Z., Sarnak, P.: The behaviour of eigenstates of arithmetic hyperbolic manifolds. Commun. Math. Phys. 161(1), 195-213 (1994)

35. Rudnick, Z., Ueberschär, H.: Statistics of wave functions for a point scatterer on the torus. Commun. Math. Phys 316(3), 763-782 (2012)

36. Rudnick, Z., Ueberschär, H.: On the eigenvalue spacing distribution for a point scatterer on the flat torus. Ann. Henri Poincaré 15(1), 1-27 (2014)

37. Šeba, P.: Wave chaos in singular quantum billiard. Phys. Rev. Lett. 64(16), 1855-1858 (1990) 
38. Šeba, P., Życzkowski, K.: Wave chaos in quantized classically nonchaotic systems. Phys. Rev. A (3) 44(6), 3457-3465 (1991)

39. Shigehara, T.: Conditions for the appearance of wave chaos in quantum singular systems with a pointlike scatterer. Phys. Rev. E 50, 4357-4370 (1994)

40. Šnirel'man, A.I.: Ergodic properties of eigenfunctions. Uspehi Mat. Nauk. 29(6(180)), 181-182 (1974)

41. Soundararajan, K.: Quantum unique ergodicity for $\mathrm{SL}_{2}(\mathbb{Z}) \backslash \mathbb{H}$. Ann. Math. (2) 172(2), 1529-1538 (2010)

42. Tanner, G.: How chaotic is the stadium billiard? A semiclassical analysis. J. Phys. A: Math. Gen. 30(8), 2863 (1997)

43. Ueberschär, H.: Quantum chaos for point scatterers on flat tori. Philos. Trans. R. Soc. Lond. Ser. A Math. Phys. Eng. Sci. 372(2007), 20120509, 12 (2014)

44. Yesha, N.: Eigenfunction statistics for a point scatterer on a three-dimensional torus. Ann. Henri Poincaré 14(7), 1801-1836 (2013)

45. Yesha, N.: Quantum ergodicity for a point scatterer on the three-dimensional torus. Annales Henri Poincaré 16(1), 1-14 (2015)

46. Zelditch, S.: Uniform distribution of eigenfunctions on compact hyperbolic surfaces. Duke Math. J. 55(4), 919-941 (1987)

Communicated by S. Zelditch 